ESAIM: M2AN 49 (2015) 1063-1084

DOI: $10.1051 / \mathrm{m} 2 \mathrm{an} / 2015005$
ESAIM: Mathematical Modelling and Numerical Analysis

www.esaim-m2an.org

\title{
SEMI HYBRID METHOD FOR HETEROGENEOUS AND ANISOTROPIC DIFFUSION PROBLEMS ON GENERAL MESHES
}

\author{
Julien COATLÉven ${ }^{1}$
}

\begin{abstract}
Symmetric, unconditionnaly coercive schemes for the discretization of heterogeneous and anisotropic diffusion problems on general, possibly nonconforming meshes are developed and studied. These schemes are a further generalization of the Hybrid Mixed Method, which allows to use a general class of consistent gradients to construct them. While the schemes are in principle hybrid, many discrete gradients or the use of correct interpolation allow to eliminate the additional face unknowns. Convergence of the approximate solutions to minimal regularity solutions is proved for general tensors and meshes. Error estimates are derived under classical regularity assumptions. Numerical results illustrate the performance of the schemes.
\end{abstract}

Mathematics Subject Classification. 65N08, 65N12, 65N15.

Received August 11, 2014. Revised January 16, 2015.

Published online June 30, 2015.

\section{INTRODUCTION}

Diffusion problems arise in many scientific fields such as heat transfer modeling, biology, plasma physics, hydrodynamics, oil reservoir simulation, etc. The classical model problem for diffusion, set on an open bounded domain $\Omega$ subset of $\mathbb{R}^{d}, d \in \mathbb{N} \backslash\{0\}$, is:

$$
-\operatorname{div}(\Lambda \nabla u)=f \quad \text { in } \Omega,
$$

which is usually complemented with homogeneous Dirichlet boundary conditions, i.e:

$$
u=0 \text { on } \partial \Omega,
$$

where $\partial \Omega=\bar{\Omega} \backslash \Omega$ is the boundary of the domain $\Omega$, assumed to be at least Lipschitz continuous. The weak solution associated to (1.1) and (1.2) is the unique $u \in H_{0}^{1}(\Omega)$ such that:

$$
\int_{\Omega} \Lambda \nabla u \nabla v=\int_{\Omega} f v \quad \forall v \text { in } H_{0}^{1}(\Omega),
$$

under the additional hypothesis that $f \in L^{2}(\Omega)$ and that $\Lambda$ is a measurable function from $\Omega$ to $\mathbb{M}_{d}(\mathbb{R})$, the set of $d \times d$ matrices, and is such that for almost every (a.e.) $\boldsymbol{x} \in \Omega, \Lambda(\boldsymbol{x})$ is symmetric, positive definite and there

Keywords and phrases. Heterogeneous diffusion problems, cell-centered methods, hybrid finite volumes, general meshes.

1 IFP Énergies nouvelles, 1 et 4 avenue de Bois-Préau, 92852 Rueil-Malmaison, France. julien.coatleven@ifpen.fr 
exists two strictly positive real numbers $\lambda_{-}, \lambda_{+}$such that for a.e. $\boldsymbol{x} \in \Omega$ and for every $\boldsymbol{\xi} \in \mathbb{R}^{d}$ :

$$
\lambda_{-}|\boldsymbol{\xi}|^{2} \leq \Lambda(\boldsymbol{x}) \boldsymbol{\xi} \cdot \boldsymbol{\xi} \leq \lambda_{+}|\boldsymbol{\xi}|^{2} .
$$

Due to the importance of diffusion problems in applications, the literature concerning their discretization is particularly rich. Classical approaches are finite difference, finite element and finite volume methods. Finite volume methods are extremely popular in industrial softwares, especially for fluid flow simulations or oil reservoir engineering. They allow to solve complex non linear physical phenomena of various natures on the same mesh, while most of the time remaining quite easy to implement.

Among finite volume methods, the most popular is undoubtledy the celebrated two-point flux approximation, which leads to compact-stencil, cell-centered, conservative and coercive schemes. To remain consistent, it requires a very strong orthogonality hypothesis on the mesh, even more difficult to satisfy in presence of anisotropy and strong heterogeneities (see [17]). However, discretization meshes are most of the time imposed by applications, and therefore distorted, nonconforming meshes must be handled.

A huge literature exists on the extension to general meshes of finite volume methods, thus we will not try to be exhaustive. For a complete review, we refer the reader to [13]. Multi-point flux approximations [1-3] are the natural generalization of the two-point scheme. They allow to construct consistent and cell-centered flux approximations on distorted meshes and lead to compact stencils, but suffer from conditional coercivity (see $[4,5]$. Recently, new finite volume methods using additional face unknowns, and thus named hybrid methods, have been introduced $[14,18,19]$. They allow to obtain both coercivity and consistency on general, possibly nonconforming meshes, for strongly heterogeneous and anisotropic tensors. The formerly introduced mimetic finite differences (see [9-12]) can be unified, in their first order formulation, with hybrid finite volumes in a generalized context (see [15]), giving birth to the class of Hybrid Mixed Methods (HMM). However, due to the additional unknowns, the resulting schemes can become expensive, in particular in a non linear setting. Notice that mimetic finite differences have recently been reinterpreted and gave birth to the very promising high order virtual element method (see [8]).

The HMM schemes share many properties with gradient schemes (see $[6,16,18,21]$ ), which, as finite elements, are based on a variational formulation of the diffusion problem rather than on flux approximation. Flux, at least in a generalized sense, can be reintroduced in this context for particular schemes (see [19]), thus emphasizing the link with finite volume approach.

Each of these methods requires a discrete approximation of the gradient. Many strongly consistent discrete approximation of this operator can be constructed on a given mesh, and there exist a huge literature about their derivation. However, few among them usually give well-posed numerical methods for diffusion problems, one of the main issues being coercivity. The coercivity and convergence of classical HMM schemes is based on the use of a particular discrete gradient operator (based on Green's formula), which naturally involves the use of face unknowns. Some schemes such as SUCCES or SUSHI (see $[19,20]$ ) allow to eliminate, at least partially, those additional unknowns through numerical interpolation, but at the expense of bad approximation properties on coarse mesh and strong heterogeneities where interpolation is used. Cell-centered Galerkin methods (see [22,23]), which are based on a discrete reconstruction operator and a discontinuous galerkin formulation of diffusion, allow a wider choice of consistent approximations of the gradient, to the expense of a more involved formulation.

Another approach for reducing the number of unknown is the hybridization technique of [24-26]: the cell unknowns are first eliminated, leading to a problem with only face unknowns, and then with a change of unknown, one can retrieve a problem based only on new cell unknowns. However, the resulting unknowns are not the original, most of the time physical, ones, and their construction requires the use of the second member of the equation, which are some of the main reasons why we do not consider this approach here.

We aim at designing symmetric, consistent and coercive methods on general nonconforming meshes, that use as few physical unknowns as possible, ideally only cell unknowns, while maintaining good approximation properties in presence of strong heterogeneities. Moreover, in the adaptive spirit of the SUSHI scheme, we also aim at providing a family of schemes that allows to use any discrete gradient operator in any part of the 
computational domain, to enhance the adaptivity of the scheme to anisotropy or heterogeneity. This will be achieved through a generalization of the construction of HMM schemes.

The outline of this paper will be as follows. In a first section, after describing meshes, notations, and the usual elements of discrete functional analysis, we start by recalling, in a slightly generalized fashion adapted to our needs, the coercivity and convergence theory of HMM methods. Then, in a second section, we explain how from any strongly consistent discrete approximation of the gradient operator, one can construct what we call semi hybrid gradients, which mainly keeps the properties of the first discrete gradient while allowing to build a coercive and convergent method, on general, nonconforming meshes, and we establish error estimates for those gradients under classical regularity assumptions. Then, we propose some possible schemes constructions, and in particular we show how one can use the ideas of the MPFA O approximation method to obtain a precise, cell-centered scheme in presence of strong heterogeneity. In the last section, we exhibit some numerical results, illustrating the good performance of these new schemes.

\section{THEORETICAL FRAMEWORK}

\subsection{Description of the meshes}

From now on, we assume that the domain $\Omega$ is a bounded polyhedral subset of $\mathbb{R}^{d}$. The mesh associated to $\Omega$ is classically defined as follows:

Definition 2.1 (General nonconforming meshes). Let $\Omega$ be a polyhedral, open bounded connected subset of $\mathbb{R}^{d}, d \in \mathbb{N} \backslash\{0\}$, and $\partial \Omega=\bar{\Omega} \backslash \Omega$ denotes its boundary. A mesh on $\Omega$, denoted by $\mathcal{M}$, is defined as a couple $\mathcal{M}=(\mathcal{T}, \mathcal{E})$ where:

1. $\mathcal{T}$ is a finite family of connected open disjoint subsets of $\Omega$ (the cells of the mesh), such that $\bar{\Omega}=\cup_{K \in \mathcal{T}} \bar{K}$. For any $K \in \mathcal{T}$, we denote by $|K|$ the $d$-dimensional Lebesgue measure of $|K|$, and we assume that $|K|>0$. We denote by $\partial K=\bar{K} \backslash K$ the boundary of $K$, and by $h_{K}$ the diameter of $K$.

2. $\mathcal{E}$ is a finite family of disjoint subsets of $\bar{\Omega}$ (the faces of the mesh) such that, for all $\sigma \in \mathcal{E}, \sigma$ is an open subset of an hyperplane of $\mathbb{R}^{d}$, and its $(d-1)$-dimensional Lebesgue measure $|\sigma|$ is assumed to be strictly positive (if $d=1$, then $|\sigma|=1$ by convention). We assume that for any $K \in \mathcal{T}$, there exist a subset $\mathcal{E}_{K}$ of $\mathcal{E}$ such that $\partial K=\cup_{\sigma \in \mathcal{E}_{K}} \bar{\sigma}$. Then, for any $\sigma \in \mathcal{E}$, we denote by $\mathcal{N}_{\sigma}=\left\{K \in \mathcal{T} \mid \sigma \in \mathcal{E}_{K}\right\}$ (the neighbors of $\sigma$ ), and assume that either $\mathcal{N}_{\sigma}$ has exactly one element, and then $\sigma \subset \partial \Omega$ (the set of these faces, called boundary faces, is denoted $\mathcal{E}_{\text {ext }}$ ), or $\mathcal{N}_{\sigma}$ has exactly two elements (the set of these faces, called interior faces, is denoted $\mathcal{E}_{\text {int }}$ ). For all $K \in \mathcal{T}$ and all $\sigma \in \mathcal{E}_{K}$, we denote by $\boldsymbol{n}_{K, \sigma}$ the unit normal vector to $\sigma$ outward to $K$, and by $\boldsymbol{x}_{\sigma}$ the barycenter of $\sigma$.

A space discretization is then defined from a mesh and discretization points associated to the mesh elements.

Definition 2.2 (Space discretization). Let $\Omega$ be a polyhedral, open bounded connected subset of $\mathbb{R}^{d}, d \in$ $\mathbb{N} \backslash\{0\}$. A space discretization of $\Omega$, denoted $\mathcal{D}$, is defined as a couple $(\mathcal{M}, \mathcal{P})$ where $\mathcal{M}$ is a mesh in the sense of Definition 2.1 and $\mathcal{P}=\left(\left(\boldsymbol{x}_{K}\right)_{K \in \mathcal{T}}\right)$ is a family of points associated to $\mathcal{M}$ and indexed by $\mathcal{T}$, such that for any $K \in \mathcal{T}, K$ is assumed to be $\boldsymbol{x}_{K}$-star shaped (which means that for any $\boldsymbol{x} \in K,\left[\boldsymbol{x}_{K}, \boldsymbol{x}\right] \subset K$ ).

- For any $K \in \mathcal{T}$ and any $\sigma \in \mathcal{E}_{K}$, we denote by $h_{K, \sigma}$ the euclidean distance between $\boldsymbol{x}_{K}$ and the hyperplane containing $\sigma$, by $d_{K, \sigma}$ the distance between $\boldsymbol{x}_{\sigma}$ and $\boldsymbol{x}_{K}$, and we assume that $h_{K, \sigma}>0$ and $d_{K, \sigma}>0$. We denote $\boldsymbol{\nu}_{K, \sigma}$ the unit vector $\boldsymbol{\nu}_{K, \sigma}=\frac{1}{d_{K, \sigma}}\left(\boldsymbol{x}_{\sigma}-\boldsymbol{x}_{K}\right)$, and remark that we have the relation:

$$
h_{K, \sigma}=d_{k, \sigma} \boldsymbol{\nu}_{K, \sigma} \cdot \boldsymbol{n}_{K, \sigma}
$$

- For any $K \in \mathcal{T}$ and any $\sigma \in \mathcal{E}_{K}$, we denote by $D_{K, \sigma}$ the cone with vertex $\boldsymbol{x}_{K}$ and basis $\sigma$, whose $d$-dimensional Lebesgue measure $\left|D_{K, \sigma}\right|$ is equal to $\frac{|\sigma| h_{K, \sigma}}{d}$. 
- We denote $h=\max _{K \in \mathcal{T}} h_{K}$ the size of the mesh, $\theta_{\mathcal{D}}=\max _{K \in \mathcal{T}, \sigma \in \mathcal{E}_{K}} \frac{d_{K, \sigma}}{h_{K, \sigma}}$ and $\theta_{\mathcal{M}}=\max _{K \in \mathcal{T}, \sigma \in \mathcal{E}_{K}} \frac{h_{K}}{h_{K, \sigma}}$.

Apart from notations, the above definition is nothing but the usual admissible finite volume mesh definition, as can be found for instance in [19]. As remarked there, this definition encompasses a wide variety of meshes, in particular it handles meshes with generalized hexahedra, i.e. with faces composed of several planar sub-faces.

\subsection{Elements of discrete functional analysis}

Classical finite volume methods only require unknowns associated with each cell $K \in \mathcal{T}$ of the mesh. Hybrid methods, as explained in the introduction, use additional unknowns associated with each face $\sigma \in \mathcal{E}$. The corresponding set of discrete unknowns is consequently:

$$
X=\left\{u_{h}=\left(\left(u_{K}\right)_{K \in \mathcal{T}},\left(u_{\sigma}\right)_{\sigma \in \mathcal{E}}\right), u_{K} \in \mathbb{R}, u_{\sigma} \in \mathbb{R}\right\} .
$$

Through numerical interpolation, some schemes such as the SUSHI schemes manage to partially eliminate the face unknowns, leading to a decomposition of $\mathcal{E}$ in two subsets:

$$
\mathcal{E}_{\mathcal{I}} \subset \mathcal{E}_{\text {int }} \quad \text { and } \quad \mathcal{E}_{\mathcal{F}}=\mathcal{E} \backslash \mathcal{E}_{\mathcal{I}},
$$

where $\mathcal{E}_{\mathcal{I}}$ corresponds to the set of faces for which we use numerical interpolation rather than a true face unknown. Thus, the practical set of discrete unknowns is the subset $X_{\mathcal{D}}$ of $X$, where:

$$
X_{\mathcal{D}}=\left\{u_{h}=\left(\left(u_{K}\right)_{K \in \mathcal{T}},\left(u_{\sigma}\right)_{\sigma \in \mathcal{E}_{\mathcal{F}}}\right), u_{K} \in \mathbb{R}, u_{\sigma} \in \mathbb{R}\right\} .
$$

A face interpolant $I_{\sigma}$ associated with a face $\sigma \in \mathcal{E}_{\mathcal{I}}$ is defined as a linear operator from $X_{\mathcal{D}}$ to $\mathbb{R}$. In order to have more compact notations, we denote $\widetilde{I}_{\sigma}\left(u_{h}\right)=I_{\sigma}\left(u_{h}\right)$ for $\sigma \in \mathcal{E}_{\mathcal{I}}$, and $\widetilde{I}_{\sigma}\left(u_{h}\right)=u_{\sigma}$ for $\sigma \in \mathcal{E}_{\mathcal{F}}$. Finally, we define $X_{\mathcal{D}, 0}$ :

$$
X_{\mathcal{D}, 0}=\left\{u_{h} \in X_{\mathcal{D}}, \mid u_{\sigma}=0 \quad \forall \sigma \in \mathcal{E}_{\text {ext }}\right\} .
$$

This set is of course designed to treat the case of homogeneous Dirichlet boundary conditions. We endow $X_{\mathcal{D}}$ with the following semi-norm:

$$
\left|u_{h}\right|_{X}^{2}=\sum_{K \in \mathcal{T}} \sum_{\sigma \in \mathcal{E}_{K}}|\sigma| h_{K, \sigma} \frac{\left|\widetilde{I}_{\sigma}\left(u_{h}\right)-u_{K}\right|^{2}}{h_{K, \sigma}^{2}},
$$

which is a norm on $X_{\mathcal{D}, 0}$, associated to the scalar product:

$$
\left(u_{h}, v_{h}\right)_{X}=\sum_{K \in \mathcal{T}} \sum_{\sigma \in \mathcal{E}_{K}}|\sigma| h_{K, \sigma} \frac{\left(\widetilde{I}_{\sigma}\left(u_{h}\right)-u_{K}\right)}{h_{K, \sigma}} \frac{\left(\widetilde{I}_{\sigma}\left(v_{h}\right)-v_{K}\right)}{h_{K, \sigma}},
$$

which makes the finite dimensional space $X_{\mathcal{D}, 0}$ a Hilbert space for this norm. It is of course intended to be a discrete version of the $H^{1}$ semi-norm.

We denote $\Pi_{\mathcal{T}}$ the operator:

$$
\begin{aligned}
\Pi_{\mathcal{T}}: X_{\mathcal{D}} & \longrightarrow L^{2}(\Omega) \\
u_{h} & \longmapsto \Pi_{\mathcal{T}}\left(u_{h}\right),
\end{aligned}
$$

where $\Pi_{\mathcal{T}}\left(u_{h}\right)(\boldsymbol{x})=u_{K}$ for a.e. $\boldsymbol{x} \in K$, for all $K \in \mathcal{T}$. For any $\varphi \in C^{0}(\bar{\Omega})$, we define the interpolant $P_{\mathcal{D}}$ by:

$$
\begin{aligned}
P_{\mathcal{D}}: C^{0}(\bar{\Omega}) & \longrightarrow X_{\mathcal{D}} \\
\varphi & \longmapsto P_{\mathcal{D}}=\left(\left(\varphi\left(\boldsymbol{x}_{K}\right)_{K \in \mathcal{T}},\left(\varphi\left(\boldsymbol{x}_{\sigma}\right)_{\sigma \in \mathcal{E}}\right)\right.\right.
\end{aligned}
$$


From the definition and Taylor's expansion, we immediately deduce that:

Lemma 2.3. For any $\varphi \in C^{1}(\bar{\Omega})$, there exists $C_{\varphi}>0$ only depending on $\varphi$ such that:

$$
\left\|\Pi_{\mathcal{T}}\left(P_{\mathcal{D}}(\varphi)\right)-\varphi\right\|_{L^{2}(\Omega)} \leq C_{\varphi} h_{\mathcal{D}}
$$

To conclude this introductory subsection, we recall two results of discrete functional analysis which will be useful in the following. All can be found in [19], to which we refer the reader for proofs.

Theorem 2.4. Let $\Omega$ be a polyhedral, open bounded connected subset of $\mathbb{R}^{d}, d \in \mathbb{N}$, and $\mathcal{M}$ be a general nonconforming mesh on $\Omega$. For any $u_{h} \in X_{\mathcal{D}}$ and any $p>1$ denote:

$$
\left|u_{h}\right|_{X, p}^{p}=\sum_{K \in \mathcal{T}} \sum_{\sigma \in \mathcal{E}_{K}}|\sigma| h_{K, \sigma}\left(\frac{\widetilde{I}_{\sigma}\left(u_{h}\right)-u_{K}}{h_{K, \sigma}}\right)^{p} .
$$

For any $1<p<d$, there exists $C>$ depending on $\Omega, d, p, \theta_{\mathcal{D}}, \theta_{\mathcal{M}}$ such that we have:

$$
\|\left.\Pi_{\mathcal{T}}\left(u_{h}\right)\right|_{L^{p^{*}}(\Omega)} \leq C\left|u_{h}\right|_{X, p} \quad \forall u_{h} \in X_{\mathcal{D}, 0}
$$

where $p^{*}=\frac{p d}{d-p}$, while for any $d \leq p<+\infty$, for any $\left.q \in\right] p,+\infty\left[\right.$, there exists $C>$ depending on $\Omega, d, p, \theta_{\mathcal{D}}, \theta_{\mathcal{M}}$ such that

$$
\|\left.\Pi_{\mathcal{T}}\left(u_{h}\right)\right|_{L^{q}(\Omega)} \leq C\left|u_{h}\right|_{X, p} \quad \forall u_{h} \in X_{\mathcal{D}, 0} .
$$

Theorem 2.5. Let $\Omega$ be a polyhedral, open bounded connected subset of $\mathbb{R}^{d}, d \in \mathbb{N} \backslash\{0\}$, and let $\mathcal{G}$ be a family of general nonconforming meshes on $\Omega$. Let $1 \leq p<+\infty$ and let $\left(u_{\mathcal{D}}\right)_{\mathcal{D} \in \mathcal{G}}$ be an associated family of functions, such that $u_{\mathcal{D}} \in X_{\mathcal{D}, 0}$ for any $\mathcal{D} \in \mathcal{G}$. Assume that there exists a constant $C>0$ such that $\left|u_{\mathcal{D}}\right|_{X, p} \leq C$ for all $\mathcal{D} \in \mathcal{G}$. Then, the family $\left(\Pi_{\mathcal{T}}\left(u_{\mathcal{D}}\right)\right)_{\mathcal{D} \in \mathcal{G}}$ is relatively compact in $L^{p}(\Omega)$, and the family of the extension by zero outside $\Omega$ of each $\Pi_{\mathcal{T}}\left(u_{\mathcal{D}}\right)$ is also relatively compact in $L^{p}\left(\mathbb{R}^{d}\right)$.

Finally, to fix vocabulary, we recall that a bilinear form $b(\cdot, \cdot)$ on a Hilbert space $H$ endowed with a norm $|\cdot|_{H}$ is said to be coercive if and only if there exists $\alpha>0$ such that for any $u \in H$, we have:

$$
\alpha|u|_{H}^{2} \leq b(u, u)
$$

\subsection{Discrete approximation}

Following the idea of hybrid schemes (or gradient schemes), we wish to approximate the variational problem $(1.3)$ on $H_{0}^{1}(\Omega)$

$$
\text { Find } u \in H_{0}^{1} \text { such that } \int_{\Omega} \Lambda \nabla u \nabla v=\int_{\Omega} f v \quad \forall v \text { in } H_{0}^{1}(\Omega),
$$

by the following variational problem on $X_{\mathcal{D}, 0}$ :

$$
\text { Find } u_{h} \in X_{\mathcal{D}, 0} \text { such that } a_{h}\left(u_{h}, v_{h}\right)+b_{h}\left(u_{h}, v_{h}\right)=l_{h}\left(v_{h}\right) \quad \forall v_{h} \in X_{\mathcal{D}, 0},
$$

where:

$$
a_{h}\left(u_{h}, v_{h}\right)=\int_{\Omega} \Lambda \nabla_{\mathcal{D}}\left(u_{h}\right) \nabla_{\mathcal{D}}\left(v_{h}\right)
$$


and

$$
l_{h}\left(v_{h}\right)=\int_{\Omega} f \Pi_{\mathcal{T}}\left(v_{h}\right),
$$

and where $b_{h}\left(u_{h}, v_{h}\right)$ is a positive, symmetric and bilinear stabilization term, designed to ensure the coercivity of the method. The operator $\nabla_{\mathcal{D}}$ is assumed to belong to a strongly consistent family of discrete gradient operators, defined as follows.

Definition 2.6 (Strongly consistent family of discrete gradient operators). Let $\Omega$ be a polyhedral, open bounded connected subset of $\mathbb{R}^{d}, d \in \mathbb{N} \backslash\{0\}$, and $\mathcal{D}$ a space discretization of $\Omega$. A discrete gradient operator $\nabla_{\mathcal{D}}$ associated to $\mathcal{D}$ is an operator from $X_{\mathcal{D}}$ to $L^{2}(\Omega)^{d}$, defined through a family of operators $\nabla_{K, \sigma}: X_{\mathcal{D}} \longrightarrow \mathbb{R}^{d}$ for all $K \in \mathcal{T}$ and all $\sigma \in \mathcal{E}_{K}$ and such that:

$$
\nabla_{\mathcal{D}}\left(u_{h}\right)=\nabla_{K, \sigma}\left(u_{h}\right) \text { a.e. in } D_{K, \sigma}, \quad \forall K \in \mathcal{T}, \forall \sigma \in \mathcal{E}_{K} .
$$

Let $\mathcal{G}$ be a family of space discretizations of $\Omega$, and let $\mathcal{Q}$ be a dense subspace of $H_{0}^{1}(\Omega)$. A strongly consistent for $(\mathcal{D})_{\mathcal{D} \in \mathcal{G}}$ on $\mathcal{Q}$ family of discrete gradient operators is a family of discrete gradient operators $\left(\nabla_{\mathcal{D}}\right)_{\mathcal{D} \in \mathcal{G}}$ such that for any $\varphi \in \mathcal{Q}$, there exists a constant $C_{\varphi}$ independent on $\mathcal{D}$ such that for any $\mathcal{D} \in \mathcal{G}$ :

$$
\left\|\nabla \varphi-\nabla_{\mathcal{D}}\left(P_{\mathcal{D}} \varphi\right)\right\|_{L^{\infty}(\Omega)^{d}} \leq C_{\varphi} h_{\mathcal{D}} .
$$

Notice that the constant $C_{\varphi}$ in the above definition can involve some bounds on parameters of the family of space discretizations, and thus the above notion of consistency depends on the considered family.

Now, we present a slight generalization of the convergence results for the HMM methods and gradient schemes which have been unified in $[6,15,16]$. From this theory, we learn that strong consistency and coercivity are not enough to obtain convergence of (2.5). This comes from the nonconformity of the approximation spaces. To obtain convergence of the schemes, an additional property, called limit conformity, is required for the discrete gradient.

Definition 2.7 (Relatively limit conforming family of discrete gradient operators). Let $\Omega$ be a polyhedral, open bounded connected subset of $\mathbb{R}^{d}, d \in \mathbb{N} \backslash 0$, let $\mathcal{Q}$ be a dense subspace of $H_{0}^{1}(\Omega)^{d}$ and let $\mathcal{G}$ be a family of space discretizations of $\Omega$. Let $\left(\nabla_{\mathcal{D}}\right)_{\mathcal{D} \in \mathcal{G}}$ be an associated family of discrete gradient operators, and let $\left(\|\cdot\|_{h_{\mathcal{D}}}\right)_{\mathcal{D} \in \mathcal{G}}$ be a family of positive linear forms. The family of gradients is said to be limit conforming for $(\mathcal{D})_{\mathcal{D} \in \mathcal{G}}$ relatively to the family of forms $\left(\|\cdot\|_{h_{\mathcal{D}}}\right)_{\mathcal{D} \in \mathcal{G}}$ if and only if, for any family of functions $\left(u_{h_{\mathcal{D}}}\right)_{\mathcal{D} \in \mathcal{G}}$ such that:

(i) $u_{h_{\mathcal{D}}} \in X_{\mathcal{D}, 0}$ for all $\mathcal{D} \in \mathcal{G}$

(ii) there exists $C>0$ independent on $n$ such that $\left\|u_{h_{\mathcal{D}}}\right\|_{h_{\mathcal{D}}} \leq C$ for all $\mathcal{D} \in \mathcal{G}$

(iii) there exists $u \in L^{2}(\Omega)$ such that $\Pi_{\mathcal{T}_{\mathcal{D}}}\left(u_{h_{\mathcal{D}}}\right) \rightarrow u$ weakly in $L^{2}(\Omega)$ when $h_{\mathcal{D}} \rightarrow 0$

we have:

$$
\int_{\Omega} \nabla_{\mathcal{D}}\left(u_{h_{\mathcal{D}}}\right) \Phi+\int_{\Omega} u \operatorname{div} \Phi \longrightarrow 0
$$

when $h_{\mathcal{D}} \rightarrow 0$, for all $\Phi \in \mathcal{Q}$. This implies that $u \in H_{0}^{1}(\Omega)$ and $\nabla_{\mathcal{D}}\left(u_{h_{\mathcal{D}}}\right)$ weakly converges to $\nabla u$ when $h_{\mathcal{D}} \rightarrow 0$.

The classical limit conformity of [21] corresponds to the particular case:

$$
\|\cdot\|_{h_{\mathcal{D}}}=\left\|\nabla_{\mathcal{D}}(\cdot)\right\|_{L^{2}(\Omega)^{d}} .
$$

The above generalization will allow us to use gradients based on MPFA approximations, whose $L^{2}$ norm is not controlled by the $|\cdot|_{X}$ norm. Further more, notice that if $(2.9)$ holds for a dense subspace $\mathcal{Q}$ of $H_{0}^{1}(\Omega)^{d}$, then 
as $\left\|\nabla_{\mathcal{D}}\left(u_{h_{\mathcal{D}}}\right)\right\|_{L^{2}(\Omega)^{d}}$ is bounded, it will hold for any other dense subspace $\widetilde{\mathcal{Q}}$ of $H_{0}^{1}(\Omega)^{d}$, which leaves us free to establish limit conformity on any dense subspace.

As an obvious adaptation of the convergence result of [21] and [6], we immediately obtain:

Theorem 2.8. Let $\Omega$ be a polyhedral, open bounded connected subset of $\mathbb{R}^{d}, d \in \mathbb{N} \backslash 0$, let $\mathcal{Q}$ be a dense subspace of $H_{0}^{1}(\Omega)$ and let $\mathcal{G}=\left(\mathcal{D}_{n}\right)_{n \in \mathbb{N}}$ be a family of space discretizations of $\Omega$ such that $h_{n}=h_{\mathcal{D}_{n}} \rightarrow 0$ when $n \rightarrow+\infty$. Assume that there exists $\theta>0$ a positive real number such that $\max \left(\theta_{\mathcal{D}}, \theta_{\mathcal{M}}\right) \leq \theta$ for all $\mathcal{D} \in \mathcal{G}$, and assume that:

(i) For any $\varphi \in \mathcal{Q}$, there exists $C_{\varphi}$ independent on $h_{n}$ such that for any $n \in \mathbb{N}$ :

$$
b_{h_{n}}\left(P_{\mathcal{D}_{n}}(\varphi), P_{\mathcal{D}_{n}}(\varphi)\right) \leq C_{\varphi} h_{n}^{2} .
$$

(ii) The family of semi hybrid gradients $\left(\nabla_{\mathcal{D}_{n}}\right)_{n \in \mathbb{N}}$ is strongly consistent for $\mathcal{G}$ on $\mathcal{Q}$ and limit conforming relatively to $\left(\|\cdot\|_{h_{n}}\right)_{n \in \mathbb{N}}$, where:

$$
\left\|v_{h_{n}}\right\|_{h_{n}}^{2}=a_{h_{n}}\left(v_{h_{n}}, v_{h_{n}}\right)+b_{h_{n}}\left(v_{h_{n}}, v_{h_{n}}\right) \quad \forall v_{h_{n}} \in X_{\mathcal{D}_{n}} .
$$

(iii) The bilinear form $a_{h_{n}}(\cdot, \cdot)+b_{h_{n}}(\cdot, \cdot)$ is coercive on $X_{n, 0}=X_{\mathcal{D}_{n}, \mathcal{I}, 0}$, i.e. there exists $\alpha>0$ independent on $h_{n}$ such that

$$
\alpha\left\|v_{h_{n}}\right\|_{X}^{2} \leq a_{h_{n}}\left(v_{h_{n}}, v_{h_{n}}\right)+b_{h_{n}}\left(v_{h_{n}}, v_{h_{n}}\right) \quad \forall v_{h_{n}} \in X_{n, 0} .
$$

Consider the sequence $\left(u_{h_{n}}\right)_{n \in \mathbb{N}}$ of solutions of the semi hybrid schemes:

$$
\text { Find } u_{h_{n}} \in X_{n, 0} \text { such that } a_{h_{n}}\left(u_{h_{n}}, v_{h_{n}}\right)+b_{h_{n}}\left(u_{h_{n}}, v_{h_{n}}\right)=l_{h_{n}}\left(v_{h_{n}}\right) \quad \forall v_{h_{n}} \in X_{n, 0} \text {. }
$$

Then for any $\mathcal{D} \in \mathcal{G}$, problem $(2.5)$ is well-posed. Moreover, denoting $u_{n}=u_{h_{n}},\left(\Pi_{\mathcal{T}_{n}}\left(u_{n}\right)\right)_{n \in \mathbb{N}}$ strongly converges to the solution $u \in H_{0}^{1}(\Omega)$ of $(1.3)$ in $L^{2}(\Omega)$ and $\left(\nabla_{\mathcal{D}_{n}}\left(u_{n}\right)\right)_{n \in \mathbb{N}}$ strongly converges to $\nabla u$ in $L^{2}(\Omega)^{d}$ when $n \rightarrow+\infty$.

We recall briefly the main steps of the proof: first, using coercivity and the discrete Poincaré's inequality of Theorem 2.4, we establish both the existence and uniqueness of the discrete solution and the boundedness of the $X_{n}$ norm of the family $\left(u_{n}\right)_{n \in \mathcal{N}}$. Then, from the compactness results of Theorem 2.5 , we deduce that, up to a subsequence, $\left(\Pi_{\mathcal{T}_{n}}\left(u_{n}\right)\right)_{n \in \mathbb{N}}$ strongly converges to some $u \in L^{2}(\Omega)$ and $\left(\nabla_{\mathcal{D}_{n}} u_{n}\right)_{n \in \mathbb{N}}$ weakly converges in $L^{2}(\Omega)$. Using the positivity of $\Lambda$, we also obtain the boundedness of $\left\|u_{n}\right\|_{h_{\mathcal{D}_{n}}}$, which gives the weak convergence of $\left(\nabla_{\mathcal{D}_{n}} u_{n}\right)_{n \in \mathbb{N}}$ to $\nabla u$. Injecting this result in the discrete formulation, and using the strong consistency of the discrete gradient on the test function $P_{\mathcal{D}_{n}}(\varphi)$, for any $\varphi \in \mathcal{Q}$, one establishes that $u$ is solution of (1.3). The strong convergence of the discrete gradient can then be established using the fact that $\left\|\nabla_{\mathcal{D}_{n}} \cdot\right\|_{L^{2}(\Omega)^{2}} \leq\|\cdot\|_{h_{\mathcal{D}_{n}}}^{2}$, and that $u$ can be approximated by a sequence of functions of $\mathcal{Q}$, strongly convergent in $H^{1}(\Omega)$.

To conclude, let us explain why contrary to classical definitions, we have not simply taken $\mathcal{Q}=C_{c}^{\infty}(\Omega)$, the space of compactly supported, smooth functions of $H_{0}^{1}(\Omega)$. Following the lines of [5], we remark that it is often more convenient to replace this space by another dense subspace of $H_{0}^{1}(\Omega)$. In particular, if:

- there exists $N$ disjoint polyhedral open sets $\Omega_{j}, 1 \leq j \leq N$, such that $\bigcup_{j=1}^{N} \bar{\Omega}_{j}=\bar{\Omega}$,

- $\Lambda \in L^{\infty}\left(\Omega, \mathbb{M}_{d}(\mathbb{R})\right)$, and for each $1 \leq j \leq N, \Lambda_{\mid \Omega_{j}} \in C^{2}\left(\overline{\Omega_{j}}, \mathbb{M}_{d}(\mathbb{R})\right)$,

- for any $\mathcal{D} \in \mathcal{G}$, for each $1 \leq j \leq N$, there exists $\mathcal{E}_{j} \subset \mathcal{E}_{\mathcal{D}}$ such that $\overline{\partial \Omega_{j}}=\bigcup_{\sigma \in \mathcal{E}_{j}} \bar{\sigma}$,

then, it is proved in [5] that the space $\mathcal{Q}_{\Lambda}$ of functions such that:

(i) $\mathcal{Q}_{\Lambda} \subset C^{0}(\bar{\Omega})$ and for any $\varphi \in \mathcal{Q}_{\Lambda}$, for each $1 \leq j \leq N, \varphi_{\mid \Omega_{j}} \in C^{2}\left(\overline{\Omega_{j}}\right)$,

(ii) for each $1 \leq i, j \leq N$ such that $\partial \Omega_{i} \cap \partial \Omega_{j}$ has dimension $d-1,\left(\nabla \varphi_{\mid \overline{\Omega_{i}}}-\nabla \varphi_{\mid \overline{\Omega_{i}}} \cdot \boldsymbol{n}_{i}\right)_{\mid \partial \Omega_{i}}=\left(\nabla \varphi_{\mid \overline{\Omega_{j}}}-\right.$ $\left.\nabla \varphi_{\mid \overline{\Omega_{j}}} \cdot \boldsymbol{n}_{j}\right)_{\mid \partial \Omega_{j}}$, i.e. the tangential derivatives are continuous, 
(iii) for each $1 \leq i, j \leq N$ such that $\partial \Omega_{i} \cap \partial \Omega_{j}$ has dimension $d-1,\left((\Lambda \nabla \varphi)_{\mid \overline{\Omega_{i}}} \cdot \boldsymbol{n}_{i}\right)_{\mid \partial \Omega_{i}}=\left((\Lambda \nabla \varphi)_{\mid \overline{\Omega_{j}}} \cdot \boldsymbol{n}_{j}\right)_{\mid \partial \Omega_{j}}$, is a dense subspace of $H_{0}^{1}(\Omega)$. This situation is extremely common in practice, and the consistency proofs for gradients and interpolants based on MPFA approaches (which we will consider as examples in the following) are readily available on $\mathcal{Q}_{\Lambda}$ but not on $C_{c}^{\infty}(\Omega)$. This is the reason why the above results are stated in this very close but more general context.

\section{THE SEMI HYBRID METHOD}

Hybrid Mixed Methods (HMM) are all based on the following discrete gradient operator, based on Green's formula (which justifies the superscript G), here written in the case without interpolation for faces:

$$
\nabla_{K, \sigma}^{G}\left(u_{h}\right)=\nabla_{K}^{G}\left(u_{h}\right)=\sum_{\sigma^{\prime} \in \mathcal{E}_{K}} \frac{\left|\sigma^{\prime}\right|}{|K|}\left(u_{\sigma^{\prime}}-u_{K}\right) \boldsymbol{n}_{K, \sigma^{\prime}} \quad \forall \sigma \in \mathcal{E}_{K}, \quad \forall K \in \mathcal{T} .
$$

In particular, the original hybrid method uses a stabilized version of this gradient, i.e.:

$$
\nabla_{K, \sigma}^{H y b r i d}\left(u_{h}\right)=\nabla_{K}^{G}\left(u_{h}\right)+\frac{\alpha}{h_{K, \sigma}}\left(\left(u_{\sigma}-u_{K}\right)-\nabla_{K}^{G}\left(u_{h}\right) \cdot\left(\boldsymbol{x}_{\sigma}-\boldsymbol{x}_{K}\right)\right) \boldsymbol{n}_{K, \sigma} .
$$

This new gradient being constructed from the Green based gradient, we will say that the Green based gradient is a "gradient seed" for the Hybrid discrete gradient. Limit conformity is ensured by the fact that the Green based gradient $\nabla_{K}^{G}\left(u_{h}\right)$ is already limit conforming, and that the stabilization term satisfies

$$
\sum_{\sigma \in \mathcal{E}_{K}} \frac{|\sigma| h_{K, \sigma}}{d} \frac{\alpha}{h_{K, \sigma}}\left(\left(u_{\sigma}-u_{K}\right)-\nabla_{K}^{G}\left(u_{h}\right) \cdot\left(\boldsymbol{x}_{\sigma}-\boldsymbol{x}_{K}\right)\right) \boldsymbol{n}_{K, \sigma}=0,
$$

which is also a consequence of this particular choice for the gradient. The constant $\alpha \neq 0$ can be changed at will: consequently, in this formulation, the "gradient seed" is fixed, while the constant is free. The Hybrid Mixed Method, by separating the discrete gradient and the stabilization term in their formulation, allows an even greater choice for $\alpha$, as it can be replaced by any positive definite matrix.

The Semi Hybrid Method we are going to present allows in the some sense the opposite freedom: we are going to show that a particular choice for the constant $\alpha$ allows to construct a limit conforming gradient whatever the choice we make on the gradient seed. We will now longer be forced to use $\nabla_{K}^{G}$ to obtain limit conformity, which will in particular allow to use gradient seeds based on cell unknowns only.

\subsection{Definition and properties}

To take into account the cases where we use gradients and interpolants adapted to the heterogeneity of the medium, let $\mathcal{Q}$ be a dense subspace of $H_{0}^{1}(\Omega)$, such that

$$
\mid \begin{aligned}
& \text { There exists } N \text { disjoint polyhedral open sets } \Omega_{j}, 1 \leq j \leq N, \text { such that } \bigcup_{j=1}^{N} \bar{\Omega}_{j}=\bar{\Omega} \\
& \mathcal{Q} \subset C^{0}(\bar{\Omega}), \text { and for each } 1 \leq j \leq N, \mathcal{Q} \subset C^{2}\left(\overline{\Omega_{j}}, \mathbb{M}_{d}(\mathbb{R})\right) .
\end{aligned}
$$

Further assume that we are given a family of space discretization $\mathcal{G}$ such that:

$$
\text { for any } \mathcal{D} \in \mathcal{G} \text {, for each } 1 \leq j \leq N \text {, there exists } \mathcal{E}_{j} \subset \mathcal{E}_{\mathcal{D}} \text { such that } \overline{\partial \Omega_{j}}=\bigcup_{\sigma \in \mathcal{E}_{j}} \bar{\sigma},
$$

i.e. the discretization is compatible with the chosen space of test functions $\mathcal{Q}$. Finally, assume that we are given an associated strongly consistent on $\mathcal{Q}$ family of discrete gradients. We denote it $\nabla_{\mathcal{D}}^{S}, S$ standing for "starting" 
or "seed" gradient. We further assume that this family is uniform over each cell, i.e. for each $K \in \mathcal{T}$, there exists an operator $\nabla_{K}^{S}$ from $X_{\mathcal{D}}$ to $\mathbb{R}^{d}$ such that:

$$
\nabla_{K, \sigma}^{S}\left(u_{h}\right)=\nabla_{K}^{S}\left(u_{h}\right) \quad \forall \sigma \in \mathcal{E}_{K}
$$

Notice that if $N=1,(3.1)$ is simply $\mathcal{Q} \subset C^{2}(\bar{\Omega})$ and (3.2) imposes nothing on the space discretization. This will be for instance the case if we work with gradient seeds and interpolations strongly consistent on $C_{c}^{\infty}(\Omega)$.

The "semi hybrid gradient" constructed from a gradient seed is defined by:

$$
\nabla_{K, \sigma}\left(u_{h}\right)=\left(\widetilde{I}_{\sigma}\left(u_{h}\right)-u_{K}\right) \frac{d \boldsymbol{n}_{K, \sigma}}{h_{K, \sigma}}+\nabla_{K}^{S}\left(u_{h}\right)-\nabla_{K}^{S}\left(u_{h}\right) \cdot\left(\boldsymbol{x}_{\sigma}-\boldsymbol{x}_{K}\right) \frac{d \boldsymbol{n}_{K, \sigma}}{h_{K, \sigma}} \quad \forall \sigma \in \mathcal{E}_{K}, \forall K \in \mathcal{T} .
$$

In other words, we make the particular choice $\alpha=d$, while we relax the choice on the gradient seed, as any strongly consistent gradient seed $\nabla_{\mathcal{D}}^{S}$ can be used, without any limit conformity requirement for it. Thus while formally similar to the original hybrid gradient, the above definition gives access to a very different family of discrete gradient operators.

Let us now explain why the choice $\alpha=d$ is particularly interesting. For any $\xi \in \mathbb{R}^{d}$, we have:

$$
\int_{K} \nabla_{\mathcal{D}}\left(u_{h}\right) \xi=\sum_{\sigma \in \mathcal{E}} \int_{\mathcal{D}_{K, \sigma}}\left(\widetilde{I}_{\sigma}\left(u_{h}\right)-u_{K}\right) \frac{d \boldsymbol{n}_{K, \sigma}}{h_{K, \sigma}} \cdot \xi+|K| \nabla_{K}^{S}\left(u_{h}\right) \cdot \xi-\sum_{\sigma \in \mathcal{E}} \int_{\mathcal{D}_{K, \sigma}} \nabla_{K}^{S}\left(u_{h}\right) \cdot\left(\boldsymbol{x}_{\sigma}-\boldsymbol{x}_{K}\right) \frac{d \boldsymbol{n}_{K, \sigma}}{h_{K, \sigma}} \cdot \xi .
$$

As the $x_{\sigma}$ are the barycenters of $\sigma$, we have the relation:

$$
\sum_{\sigma \in \mathcal{E}_{K}} \frac{|\sigma|}{|K|}\left(\boldsymbol{x}_{\sigma}-\boldsymbol{x}_{K}\right) \boldsymbol{n}_{K, \sigma}^{T}=I d .
$$

Thus, using $\left|\mathcal{D}_{K, \sigma}\right|=\frac{|\sigma| h_{K, \sigma}}{d}$, the last two term cancel each other, and we obtain:

$$
\int_{K} \nabla_{\mathcal{D}}\left(u_{h}\right) \xi=\sum_{\sigma \in \mathcal{E}}|\sigma|\left(\widetilde{I}_{\sigma}\left(u_{h}\right)-u_{K}\right) \boldsymbol{n}_{K, \sigma} \cdot \xi
$$

This relation is the key to establish the relative limit conformity of the semi hybrid gradient, for any gradient seed:

Lemma 3.1. Let $\Omega$ be a polyhedral, open bounded connected subset of $\mathbb{R}^{d}, d \in \mathbb{N} \backslash 0$, and let $\mathcal{G}$ be a family of space discretizations of $\Omega$. Assume that there exists $\theta>0$ such that $\max \left(\theta_{\mathcal{D}}, \theta_{\mathcal{M}}\right) \leq \theta$ for all $\mathcal{D} \in \mathcal{G}$. Then the family of semi hybrid gradients $\left(\nabla_{\mathcal{D}}\right)_{\mathcal{D} \in \mathcal{G}}$ is limit conforming on $(\mathcal{D})_{\mathcal{D} \in \mathcal{G}}$ relatively to $\|\cdot\|_{\tilde{X}}$, where we have denoted:

$$
\left\|u_{h}\right\|_{\tilde{X}}^{2}=\left\|\nabla_{\mathcal{D}}\left(u_{h}\right)\right\|_{L^{2}(\Omega)^{d}}^{2}+\left|u_{h}\right|_{X}^{2} .
$$

Proof. Let $\left(u_{h_{\mathcal{D}}}\right)_{\mathcal{D} \in \mathcal{G}}$ be a family of functions, such that:

(i) $u_{h_{\mathcal{D}}} \in X_{\mathcal{D}, 0}$ for all $\mathcal{D} \in \mathcal{G}$.

(ii) There exists $C>0$ independent on $n$ such that $\left\|u_{h}\right\|_{\tilde{X}} \leq C$ for all $\mathcal{D} \in \mathcal{G}$.

(iii) There exists $u \in L^{2}(\Omega)$ such that $\Pi_{\mathcal{T}_{\mathcal{D}}}\left(u_{h_{\mathcal{D}}}\right) \rightarrow u$ weakly in $L^{2}(\Omega)$ when $h_{\mathcal{D}} \rightarrow 0$.

First of all, we have, for any $\Phi \in C_{c}^{\infty}(\Omega)^{d}$

$$
\int_{\Omega} \nabla_{\mathcal{D}}\left(u_{h}\right) \Phi=\sum_{K \in \mathcal{T}} \int_{K} \nabla_{\mathcal{D}}\left(u_{h}\right) \Phi_{K}+\sum_{K \in \mathcal{T}} \int_{K} \nabla_{\mathcal{D}}\left(u_{h}\right)\left(\Phi_{K}-\Phi\right),
$$


where we have denoted $\Phi_{K}=\frac{1}{|K|} \int_{K} \Phi$. For some $C_{\Phi}$ depending only on $\Phi$, we have $\left|\left(\Phi_{K}-\Phi\right)\right| \leq C_{\Phi} h_{\mathcal{D}}$ and thus, using Cauchy-Schwarz inequality, the second term is bounded by:

$$
\sum_{K \in \mathcal{T}} \int_{K} \nabla_{\mathcal{D}}\left(u_{h}\right)\left(\Phi_{K}-\Phi\right) \leq C_{\Phi} h_{\mathcal{D}}|\operatorname{supp} \Phi|^{1 / 2}\left\|\nabla_{\mathcal{D}}\left(u_{h}\right)\right\|_{L^{2}(\Omega)^{d}}
$$

Then, notice that

$$
\int_{\Omega} \Pi_{\mathcal{T}}\left(u_{h_{\mathcal{D}}}\right) \operatorname{div} \Phi=\sum_{K \in \mathcal{T}} u_{K} \sum_{\sigma \in \mathcal{E}_{K}} \int_{\sigma} \Phi \cdot \boldsymbol{n}_{K, \sigma} .
$$

Denoting $\Phi_{\sigma}=\frac{1}{|\sigma|} \int_{\sigma} \Phi$, we get:

$$
\int_{\Omega} \Pi_{\mathcal{T}}\left(u_{h_{\mathcal{D}}}\right) \operatorname{div} \Phi=\sum_{K \in \mathcal{T}} u_{K} \sum_{\sigma \in \mathcal{E}_{K}}|\sigma| \Phi_{\sigma} \cdot \boldsymbol{n}_{K, \sigma} .
$$

As $\boldsymbol{n}_{K, \sigma}+\boldsymbol{n}_{L, \sigma}=0$ and as $\Phi$ is compactly supported in $\Omega$, and using (3.5):

$$
\begin{aligned}
\int_{\Omega} \Pi_{\mathcal{T}}\left(u_{h_{\mathcal{D}}}\right) \operatorname{div} \Phi= & \sum_{K \in \mathcal{T}} \sum_{\sigma \in \mathcal{E}_{K}}|\sigma|\left(u_{K}-\widetilde{I}_{\sigma}\left(u_{h}\right)\right) \Phi_{\sigma} \cdot \boldsymbol{n}_{K, \sigma} \\
= & \sum_{K \in \mathcal{T}} \int_{K}\left(\sum_{\sigma \in \mathcal{E}_{K}} \frac{|\sigma|}{|K|}\left(u_{K}-\widetilde{I}_{\sigma}\left(u_{h}\right)\right) \boldsymbol{n}_{K, \sigma}\right) \cdot \Phi_{K} \\
& +\sum_{K \in \mathcal{T}} \sum_{\sigma \in \mathcal{E}_{K}}|\sigma|\left(u_{K}-\widetilde{I}_{\sigma}\left(u_{h}\right)\right)\left(\Phi_{\sigma}-\Phi_{K}\right) \cdot \boldsymbol{n}_{K, \sigma} \\
= & -\sum_{K \in \mathcal{T}} \int_{K} \nabla_{\mathcal{D}}\left(u_{h_{\mathcal{D}}}\right) \cdot \Phi_{K}+\sum_{K \in \mathcal{T}} \sum_{\sigma \in \mathcal{E}_{K}}|\sigma|\left(u_{K}-\widetilde{I}_{\sigma}\left(u_{h}\right)\right)\left(\Phi_{\sigma}-\Phi_{K}\right) \cdot \boldsymbol{n}_{K, \sigma} .
\end{aligned}
$$

The last term gives:

$$
\begin{aligned}
& \left|\sum_{K \in \mathcal{T}} \sum_{\sigma \in \mathcal{E}_{K}}\right| \sigma\left|\left(u_{K}-\widetilde{I}_{\sigma}\left(u_{h}\right)\right)\left(\Phi_{\sigma}-\Phi_{K}\right) \cdot \boldsymbol{n}_{K, \sigma}\right| \\
\leq h_{\mathcal{D}} C_{\Phi} & \left(\sum_{K \in \mathcal{T}, K \cap \operatorname{supp} \varphi \neq \emptyset} \sum_{\sigma \in \mathcal{E}_{K}} \frac{|\sigma| h_{K, \sigma}^{2}}{h_{K, \sigma}}\right)^{1 / 2}\left(\sum_{K \in \mathcal{T}} \sum_{\sigma \in \mathcal{E}_{K}}|\sigma| h_{K, \sigma} \frac{\left|u_{K}-\widetilde{I}_{\sigma}\left(u_{h}\right)\right|^{2}}{h_{K, \sigma}^{2}}\right)^{1 / 2} \\
\leq d^{1 / 2} C_{\Phi} h_{\mathcal{D}}|\operatorname{supp} \Phi|^{1 / 2}\left|u_{h_{\mathcal{D}}}\right|_{X} . &
\end{aligned}
$$

Collecting these results, using hypothesis (ii) and (iii) and letting $h_{\mathcal{D}}$ go to zero, we obtain (2.9), which concludes the proof.

Remark 3.2. Another interesting choice for $\alpha$ is $\alpha=1$. The corresponding discrete gradient $\widetilde{\nabla}_{K, \sigma}$ would indeed satisfy:

$$
\widetilde{\nabla}_{K, \sigma}\left(u_{h}\right) \cdot \boldsymbol{\nu}_{K, \sigma}=\frac{\widetilde{I}_{\sigma}\left(u_{h}\right)-u_{K}}{d_{K, \sigma}} \text { for } \sigma \in \mathcal{E}_{K}
$$

and thus, for some $C>$ independent on $h_{\mathcal{D}}$ :

$$
\int_{\Omega} \Lambda \widetilde{\nabla}_{\mathcal{D}}\left(u_{h}\right) \widetilde{\nabla}_{\mathcal{D}}\left(u_{h}\right) \geq C \lambda_{-}\left|u_{h}\right|_{X}^{2}
$$

This gives coercivity without any stabilization term. However, limit conformity is not guaranteed, which limits the gradient seeds that can be used, contrary to the choice $\alpha=d$. 
In addition to their limit conforming property, the semi hybrid gradients should also be strongly consistent. As noticed in $[19,20]$, face interpolants have to be at least order 2 consistent to obtain consistency of the resulting gradient, i.e. for any $\varphi \in \mathcal{Q}$, there exists $C_{\varphi}>0$ only depending on $\varphi$ such that for any $\sigma \in \mathcal{E}_{\mathcal{I}}$ :

$$
\left|I_{\sigma}\left(P_{\mathcal{D}} \varphi\right)-\varphi\left(\boldsymbol{x}_{\sigma}\right)\right| \leq C_{\varphi} h_{K, \sigma} h_{\mathcal{D}}
$$

Lemma 3.3 (Strong consistency of semi hybrid gradients). Let $\Omega$ be a polyhedral, open bounded connected subset of $\mathbb{R}^{d}, d \in \mathbb{N} \backslash 0$, let $\mathcal{Q}$ be a dense subset of $H_{0}^{1}(\Omega)$ satisfying (3.1) and let $\mathcal{G}$ be a family of space discretizations of $\Omega$ satisfying (3.2). Let $\left(\nabla_{\mathcal{D}}^{S}\right)_{\mathcal{D} \in \mathcal{G}}$ be an associated family of discrete gradient seeds. Further assume that for any $\mathcal{D} \in \mathcal{G}$ and any $\sigma \in \mathcal{E}_{\mathcal{I}},(3.6)$ holds. Assume that there exists $\theta>0$ such that $\max \left(\theta_{\mathcal{D}}, \theta_{\mathcal{M}}\right) \leq$ $\theta$ for all $\mathcal{D} \in \mathcal{G}$. Then the family of semi hybrid gradients constructed from those gradient seeds is strongly consistent on $\mathcal{Q}$.

Proof. Let $\varphi$ belongs to $\mathcal{Q}$. Then, for any $K \in \mathcal{T}$, and any $\sigma \in \mathcal{E}$ :

$$
\begin{aligned}
\left|\nabla_{K, \sigma} P_{\mathcal{D}}(\varphi)-\nabla \varphi\left(\boldsymbol{x}_{K}\right)\right| \leq & \left|\nabla_{K}^{S} P_{\mathcal{D}}(\varphi)-\nabla \varphi\left(\boldsymbol{x}_{K}\right)\right| \\
& +\left|\frac{\widetilde{I}_{\sigma}\left(P_{\mathcal{D}}(\varphi)\right)-\varphi\left(\boldsymbol{x}_{K}\right)}{h_{K, \sigma}} \boldsymbol{n}_{K, \sigma}-\nabla_{K}^{S} P_{\mathcal{D}}(\varphi) \cdot \boldsymbol{\nu}_{K, \sigma} \frac{d_{K, \sigma}}{h_{K, \sigma}} \boldsymbol{n}_{K, \sigma}\right| .
\end{aligned}
$$

We have $\left|\nabla_{K}^{S} P_{\mathcal{D}}(\varphi)-\nabla \varphi\left(\boldsymbol{x}_{K}\right)\right| \leq C_{\varphi} h_{\mathcal{D}}$. For the second term, notice that

$$
\begin{gathered}
\left|\frac{\widetilde{I}_{\sigma}\left(P_{\mathcal{D}}(\varphi)\right)-\varphi\left(\boldsymbol{x}_{K}\right)}{h_{K, \sigma}} \boldsymbol{n}_{K, \sigma}-\nabla_{K}^{S} P_{\mathcal{D}}(\varphi) \cdot \boldsymbol{\nu}_{K, \sigma} \frac{d_{K, \sigma}}{h_{K, \sigma}} \boldsymbol{n}_{K, \sigma}\right| \leq \\
\left|\frac{\widetilde{I}_{\sigma}\left(P_{\mathcal{D}}(\varphi)\right)-\varphi\left(\boldsymbol{x}_{K}\right)}{h_{K, \sigma}}-\nabla \varphi\left(\boldsymbol{x}_{K}\right) \cdot \frac{d_{K, \sigma}}{h_{K, \sigma}} \boldsymbol{\nu}_{K, \sigma}\right|+\left|\nabla \varphi\left(\boldsymbol{x}_{K}\right) \cdot \frac{d_{K, \sigma}}{h_{K, \sigma}} \boldsymbol{\nu}_{K, \sigma}-\nabla_{K}^{S} P_{\mathcal{D}}(\varphi) \cdot \boldsymbol{\nu}_{K, \sigma} \frac{d_{K, \sigma}}{h_{K, \sigma}}\right| .
\end{gathered}
$$

Using the hypothesis of strong consistency of gradient seeds, the consistency hypothesis on the interpolants, the compatibility hypothesis (3.2) and the regularity of $\varphi$ in each $\Omega_{j}$, we obtain that for some $C_{\varphi}>0$ depending only on $\varphi$ :

$$
\left|\frac{\widetilde{I}_{\sigma}\left(P_{\mathcal{D}}(\varphi)\right)-\varphi\left(\boldsymbol{x}_{K}\right)}{h_{K, \sigma}} \boldsymbol{n}_{K, \sigma}-\nabla_{K}^{S} P_{\mathcal{D}}(\varphi) \cdot \boldsymbol{\nu}_{K, \sigma} \frac{d_{K, \sigma}}{h_{K, \sigma}} \boldsymbol{n}_{K, \sigma}\right| \leq \theta C_{\varphi} h_{\mathcal{D}},
$$

which leads to the conclusion.

Finally, it only remains to ensure the coercivity of the method, by choosing an appropriate stabilization term. We use the same term than for HMM schemes, but with the semi hybrid gradient replacing the classical hybrid gradient:

$$
b_{h}\left(u_{h}, v_{h}\right)=\sum_{K \in \mathcal{T}} \sum_{\sigma \in \mathcal{E}_{K}} \frac{|\sigma| h_{K, \sigma}}{d} S_{K, \sigma} R_{K, \sigma}\left(u_{h}\right) R_{K, \sigma}\left(v_{h}\right)
$$

where

$$
R_{K, \sigma}\left(u_{h}\right)=\left(\frac{\widetilde{I}_{\sigma}\left(u_{h}\right)-u_{K}}{h_{K, \sigma}}-\nabla_{K, \sigma}\left(u_{h}\right) \cdot \frac{\left(\boldsymbol{x}_{\sigma}-\boldsymbol{x}_{K}\right)}{h_{K, \sigma}}\right) \boldsymbol{n}_{K, \sigma} \quad \forall \sigma \in \mathcal{E}_{K}, \forall K \in \mathcal{T},
$$

and $S_{K, \sigma}$ is a family of symmetric, positive definite matrices such that for any $\xi \in \mathbb{R}^{d}, S_{K, \sigma} \xi \cdot \xi \geq \beta_{-}|\xi|^{2}$ for some $\beta_{-}>0$. Consistency, when interpolants are order 2 consistent, can be obtained exactly as in the above proof, which is why we do not detail it here. Coercivity then comes from the inequality:

$$
(a-b)^{2} \geq \frac{\mu}{1+\mu} a^{2}-\mu b^{2}, \quad \forall a, b \in \mathbb{R}, \forall \mu>-1 .
$$


Applying this to $S_{K, \sigma} R_{K, \sigma}\left(u_{h}\right) R_{K, \sigma}\left(u_{h}\right)$, we obtain, using the notation $\widetilde{I}$ again:

$$
\begin{aligned}
S_{K, \sigma} R_{K, \sigma}\left(u_{h}\right) R_{K, \sigma}\left(v_{h}\right) & \geq \beta_{-}\left(\frac{\widetilde{I}_{\sigma}\left(u_{h}\right)-u_{K}}{h_{K, \sigma}}-\nabla_{K, \sigma}\left(u_{h}\right) \cdot \frac{\boldsymbol{x}_{\sigma}-\boldsymbol{x}_{K}}{h_{K, \sigma}}\right)^{2} . \\
& \geq \frac{\beta_{-} \mu}{1+\mu}\left(\frac{\widetilde{I}_{\sigma}\left(u_{h}\right)-u_{K}}{h_{K, \sigma}}\right)^{2}-\beta_{-} \mu\left|\nabla_{K, \sigma}\left(u_{h}\right)\right|^{2}\left(\frac{d_{K, \sigma}}{h_{K, \sigma}}\right)^{2} .
\end{aligned}
$$

Thus, we get:

$$
a_{h}\left(u_{h}, u_{h}\right)+b_{h}\left(u_{h}, u_{h}\right) \geq \sum_{K \in \mathcal{T}} \sum_{\sigma \in \mathcal{E}_{K}} \frac{|\sigma| h_{K, \sigma}}{d}\left(\left(\lambda_{-}-\beta_{-} \mu \theta^{2}\right)\left|\nabla_{K, \sigma}\left(u_{h}\right)\right|^{2}+\frac{\beta_{-} \mu}{1+\mu}\left(\frac{\widetilde{I}_{\sigma}\left(u_{h}\right)-u_{K}}{h_{K, \sigma}}\right)^{2}\right) .
$$

Taking $\mu$ such that $\mu<\frac{\lambda_{-}}{\beta_{-} \theta^{2}}$, we obtain that there exists $\alpha>0$ such that:

$$
a_{h}\left(u_{h}, u_{h}\right)+b_{h}\left(u_{h}, u_{h}\right) \geq \alpha\left(\left\|\nabla_{\mathcal{D}}\left(u_{h}\right)\right\|_{L^{2}(\Omega)^{d}}^{2}+\left|u_{h}\right|_{X}^{2}\right) .
$$

This result not only establishes the coercivity of the method but also, using Lemma 2.3, the limit conformity of the semi hybrid gradient relatively to the $\|\cdot\|_{h_{\mathcal{D}}}$ norm. Combining all these results, we see that all hypothesis of Theorem 2.8 are satisfied, and thus, the semi hybrid method provides convergent approximations of minimal regularity solutions. We also see that choosing the matrix $S_{K, \sigma}$ such that $\beta_{-} \leq \lambda_{-}$should improve the coercivity constant of the scheme. A natural choice is thus $S_{K, \sigma}=\Lambda_{K, \sigma}$, with $\Lambda_{K, \sigma}=\frac{1}{\mid D_{K, \sigma}} \int_{D_{K, \sigma}} \Lambda$, which is what we use in practice, and what we will assume from now on.

\subsection{Elimination of face unknowns}

The variational problem (2.5) can be rewritten:

$$
\sum_{K \in \mathcal{T}} B_{K}\left(u_{h}\right) v_{K}+\sum_{\sigma \in \mathcal{E} \cap \mathcal{E}_{\mathcal{F}}} \sum_{K \in \mathcal{N}_{\sigma}} F_{K, \sigma}\left(u_{h}\right) v_{\sigma}=\sum_{K \in \mathcal{T}} \int_{K} f v_{K} \quad \forall v_{h} \in X_{\mathcal{D}, 0} .
$$

Remark that we consequently have:

$$
B_{K}\left(u_{h}\right)=\int_{K} f \quad \forall K \in \mathcal{T}, \forall \sigma \in \mathcal{E}_{K},
$$

and

$$
F_{K, \sigma}\left(u_{h}\right)+F_{L, \sigma}\left(u_{h}\right)=0 \quad \forall \sigma \in \mathcal{E} \cap \mathcal{E}_{\mathcal{F}}, \mathcal{N}_{\sigma}=\{K, L\} .
$$

Assume now that in each cell $K$, all the gradient seed only depend on cell values (i.e. on $u_{\mathcal{T}}$ ), then (3.9) rewrites:

$$
F_{K, \sigma}\left(u_{\mathcal{T}}, u_{\sigma}\right)+F_{L, \sigma}\left(u_{\mathcal{T}}, u_{\sigma}\right)=0 \quad \forall \sigma \in \mathcal{E} \cap \mathcal{E}_{\mathcal{F}}, \mathcal{N}_{\sigma}=\{K, L\}
$$

The coercivity of the scheme implies that this linear equation is uniquely solvable with respect to $u_{\sigma}$, thus we can write:

$$
u_{\sigma}=\mathcal{F}_{\sigma}\left(u_{\mathcal{T}}\right) .
$$

The function $\mathcal{F}_{\sigma}$ can even be explicited in this case. Indeed, denoting $\lambda_{K, \sigma}=\Lambda_{K, \sigma} \boldsymbol{n}_{K, \sigma} \cdot \boldsymbol{n}_{K, \sigma}$, and with the choice $S_{K, \sigma}=\Lambda_{K, \sigma}$ for the stabilization term, we have

$$
\begin{aligned}
F_{K, \sigma}\left(u_{\mathcal{T}}, u_{\sigma}\right)= & \frac{d|\sigma| \lambda_{K, \sigma}}{h_{K, \sigma}}\left(\left(u_{\sigma}-u_{K}\right)-\nabla_{K}^{S}\left(u_{\mathcal{T}}\right) \cdot\left(\boldsymbol{x}_{\sigma}-\boldsymbol{x}_{K}\right)\right)+|\sigma| \Lambda_{K, \sigma} \nabla_{K}^{S}\left(u_{\mathcal{T}}\right) \cdot \boldsymbol{n}_{K, \sigma} \\
& +\frac{|\sigma| \lambda_{K, \sigma}}{d h_{K, \sigma}}\left(1-d \boldsymbol{n}_{K, \sigma} \cdot \frac{\left(\boldsymbol{x}_{\sigma}-\boldsymbol{x}_{K}\right)}{h_{K, \sigma}}\right)^{2}\left(\left(u_{\sigma}-u_{K}\right)-\nabla_{K}^{S}\left(u_{\mathcal{T}}\right) \cdot\left(\boldsymbol{x}_{\sigma}-\boldsymbol{x}_{K}\right)\right),
\end{aligned}
$$


and thus, denoting:

$$
\omega_{K, \sigma}=\frac{d|\sigma| \lambda_{K, \sigma}}{h_{K, \sigma}}+\frac{|\sigma| \lambda_{K, \sigma}}{d h_{K, \sigma}}\left(1-d \boldsymbol{n}_{K, \sigma} \cdot \frac{\left(\boldsymbol{x}_{\sigma}-\boldsymbol{x}_{K}\right)}{h_{K, \sigma}}\right)^{2}
$$

we obtain:

$$
u_{\sigma}=\frac{\omega_{K, \sigma} u_{K}+\omega_{L, \sigma} u_{L}}{\omega_{K, \sigma}+\omega_{L, \sigma}}+\nabla_{K}^{S}\left(u_{\mathcal{T}}\right) \cdot \frac{\omega_{K, \sigma}\left(\boldsymbol{x}_{\sigma}-\boldsymbol{x}_{K}\right)-|\sigma| \Lambda_{K, \sigma}^{T} \boldsymbol{n}_{K, \sigma}}{\omega_{K, \sigma}+\omega_{L, \sigma}}+\nabla_{L}^{S}\left(u_{\mathcal{T}}\right) \cdot \frac{\omega_{L, \sigma}\left(\boldsymbol{x}_{\sigma}-\boldsymbol{x}_{L}\right)-|\sigma| \Lambda_{L, \sigma}^{T} \boldsymbol{n}_{L, \sigma}}{\omega_{K, \sigma}+\omega_{L, \sigma}} .
$$

The scheme reduces to:

$$
B_{K}\left(u_{\mathcal{T}},\left(\mathcal{F}_{\sigma}\left(u_{\mathcal{T}}\right)\right)_{\sigma \in \mathcal{E} \cap \mathcal{E}_{\mathcal{F}}}\right)=\int_{K} f \quad \forall K \in \mathcal{T}
$$

i.e. a scheme using only cell unknowns. It means that semi hybrid schemes very often allow to eliminate face unknowns, which is the reason why they are only "semi" hybrid, whereas they are initially constructed as full hybrid schemes. Apart from the ability to use the discrete gradient of one's choice, this elimination is one of the principal interests of those schemes (the situation is in fact the same that for the hybrid scheme described in [7], but with a much larger choice of gradients here).

\subsection{Error estimates}

We now derive error estimates:

Theorem 3.4. Under the same hypothesis than Theorem 2.8 , further assume that $\mathcal{Q} \subset C^{2}(\bar{\Omega})$, that (3.6) holds, and that $\Lambda \in C^{1}\left(\bar{\Omega}, \mathbb{M}_{d}(\mathbb{R})\right)$ and $u \in C^{2}(\bar{\Omega})$. Then, there exists $C>0$ independent on $h_{\mathcal{D}}$ such that:

$$
\begin{gathered}
\left\|\nabla u-\nabla_{\mathcal{D}}\left(u_{h_{\mathcal{D}}}\right)\right\|_{L^{2}(\Omega)^{d}} \leq C h_{\mathcal{D}}, \\
\left\|u-\Pi_{\mathcal{T}}\left(u_{h_{\mathcal{D}}}\right)\right\|_{L^{2}(\Omega)} \leq C h_{\mathcal{D}} .
\end{gathered}
$$

Proof. As $\Lambda \in C^{1}\left(\bar{\Omega}, \mathbb{M}_{d}(\mathbb{R})\right)$ and $u \in C^{2}(\bar{\Omega})$, for any $v_{h_{\mathcal{D}}} \in X_{\mathcal{D}, 0}$, we can write that:

$$
\begin{aligned}
\int_{\Omega} f \Pi_{\mathcal{T}}\left(v_{h_{\mathcal{D}}}\right) & =-\int_{\Omega} \operatorname{div}(\Lambda \nabla u) \Pi_{\mathcal{T}}\left(v_{h_{\mathcal{D}}}\right)=-\sum_{K \in \mathcal{T}} v_{K} \int_{K} \operatorname{div}(\Lambda \nabla u) \\
& =-\sum_{K \in \mathcal{T}} v_{K} \sum_{\sigma \in \mathcal{E}_{K}} \int_{\sigma} \Lambda \nabla u \cdot \boldsymbol{n}_{K, \sigma}=-\sum_{K \in \mathcal{T}} \sum_{\sigma \in \mathcal{E}_{K}}\left(v_{K}-\widetilde{I}_{\sigma}\left(v_{h_{\mathcal{D}}}\right)\right) \int_{\sigma} \Lambda \nabla u \cdot \boldsymbol{n}_{K, \sigma} .
\end{aligned}
$$

Proceeding as in the proof of Lemma 2.3, we get that:

$$
\int_{\Omega} f \Pi_{\mathcal{T}}\left(v_{h_{\mathcal{D}}}\right)=\sum_{K \in \mathcal{T}} \int_{K} \nabla_{\mathcal{D}}\left(v_{h_{\mathcal{D}}}\right) \cdot \Lambda \nabla u\left(\boldsymbol{x}_{K}\right)-\sum_{K \in \mathcal{T}} \sum_{\sigma \in \mathcal{E}_{K}}\left(v_{K}-\widetilde{I}_{\sigma}\left(v_{h_{\mathcal{D}}}\right)\right) \int_{\sigma}\left(\Lambda \nabla u-(\Lambda \nabla u)\left(\boldsymbol{x}_{K}\right)\right) \cdot \boldsymbol{n}_{K, \sigma}
$$

Thus, using the fact that $u_{h_{\mathcal{D}}}$ is solution of (2.5), we get:

$$
\begin{aligned}
a_{h_{\mathcal{D}}}\left(u_{h_{\mathcal{D}}}, v_{h_{\mathcal{D}}}\right)+b_{h_{\mathcal{D}}}\left(u_{h_{\mathcal{D}}}, v_{h_{\mathcal{D}}}\right)= & \int_{\Omega} \Lambda \nabla u \nabla_{\mathcal{D}}\left(v_{h_{\mathcal{D}}}\right)+\sum_{K \in \mathcal{T}} \int_{K}\left((\Lambda \nabla u)\left(\boldsymbol{x}_{K}\right)-\Lambda \nabla u\right) \nabla_{\mathcal{D}}\left(v_{h_{\mathcal{D}}}\right) \\
& +\sum_{K \in \mathcal{T}} \sum_{\sigma \in \mathcal{E}_{K}}\left(\widetilde{I}_{\sigma}\left(v_{h_{\mathcal{D}}}\right)-v_{K}\right) \int_{\sigma}\left(\Lambda \nabla u-(\Lambda \nabla u)\left(\boldsymbol{x}_{K}\right)\right) \cdot \boldsymbol{n}_{K, \sigma} .
\end{aligned}
$$


Consequently, we have that:

$$
\begin{array}{r}
a_{h_{\mathcal{D}}}\left(P_{\mathcal{D}}(u)-u_{h_{\mathcal{D}}}, v_{h_{\mathcal{D}}}\right)+b_{h_{\mathcal{D}}}\left(P_{\mathcal{D}}(u)-u_{h_{\mathcal{D}}}, v_{h_{\mathcal{D}}}\right)=\int_{\Omega} \Lambda\left(\nabla_{\mathcal{D}}\left(P_{\mathcal{D}}(u)-\nabla u\right) \nabla_{\mathcal{D}}\left(v_{h_{\mathcal{D}}}\right)\right. \\
+b_{h_{\mathcal{D}}}\left(P_{\mathcal{D}}(u), v_{h_{\mathcal{D}}}\right)-\sum_{K \in \mathcal{T}} \sum_{\sigma \in \mathcal{E}_{K}} \int_{D_{K, \sigma}}\left((\Lambda \nabla u)\left(\boldsymbol{x}_{K}\right)-\Lambda \nabla u\right) \nabla_{\mathcal{D}}\left(v_{h_{\mathcal{D}}}\right) \\
-\sum_{K \in \mathcal{T}} \sum_{\sigma \in \mathcal{E}_{K}}\left(\widetilde{I}_{\sigma}\left(v_{h_{\mathcal{D}}}\right)-v_{K}\right) \int_{\sigma}\left(\Lambda \nabla u-(\Lambda \nabla u)\left(\boldsymbol{x}_{K}\right)\right) \cdot \boldsymbol{n}_{K, \sigma} .
\end{array}
$$

With obvious notations, we denote $a_{h_{\mathcal{D}}}\left(P_{\mathcal{D}}(u)-u_{h_{\mathcal{D}}}, v_{h_{\mathcal{D}}}\right)+b_{h_{\mathcal{D}}}\left(P_{\mathcal{D}}(u)-u_{h_{\mathcal{D}}}, v_{h_{\mathcal{D}}}\right)=T_{1}+T_{2}+T_{3}+T_{4}$ and recall that:

$$
\left\|v_{h_{\mathcal{D}}}\right\|_{h_{\mathcal{D}}}^{2}=\left\|\nabla_{\mathcal{D}}\left(v_{h_{\mathcal{D}}}\right)\right\|_{L^{2}(\Omega)^{d}}^{2}+b_{h_{\mathcal{D}}}\left(v_{h_{\mathcal{D}}}, v_{h_{\mathcal{D}}}\right) .
$$

Now, we bound each term of the right hand side:

$$
T_{1} \leq \lambda_{+}\left\|\nabla_{\mathcal{D}}\left(P_{\mathcal{D}}(u)\right)-\nabla u\right\|_{L^{2}(\Omega)^{d}}\left\|\nabla_{\mathcal{D}}\left(v_{h_{\mathcal{D}}}\right)\right\|_{L^{2}(\Omega)^{d}} \leq \lambda_{+} h_{\mathcal{D}} C_{u}|\Omega|^{\frac{1}{2}}\left\|v_{h_{\mathcal{D}}}\right\|_{h_{\mathcal{D}}},
$$

where $C_{u}$ is a constant depending on $u$ and $\Omega$ (more precisely of the $C^{2}$-norm of $u$ ). Using Cauchy-Schwarz inequality and the strong consistency of the stabilization term, we get that

$$
T_{2} \leq b_{h_{\mathcal{D}}}\left(P_{\mathcal{D}}(u), P_{\mathcal{D}}(u)\right)^{\frac{1}{2}} b_{h_{\mathcal{D}}}\left(v_{h_{\mathcal{D}}}, v_{h_{\mathcal{D}}}\right)^{\frac{1}{2}} \leq C_{u} \lambda_{+} h_{\mathcal{D}}\left\|v_{h_{\mathcal{D}}}\right\|_{h_{\mathcal{D}}} .
$$

Then

$$
T_{3} \leq \lambda_{+} C_{\Lambda, u} h_{\mathcal{D}} \int_{\Omega}\left|\nabla_{\mathcal{D}} v_{h_{\mathcal{D}}}\right| \leq \lambda_{+} C_{\Lambda, u} h_{\mathcal{D}}|\Omega|^{\frac{1}{2}}|| \nabla_{\mathcal{D}} v_{h_{\mathcal{D}}}\left\|_{L^{2}(\Omega)^{d}} \leq \lambda_{+} C_{\Lambda, u} h_{\mathcal{D}}|\Omega|^{\frac{1}{2}}\right\| v_{h_{\mathcal{D}}} \|_{h_{\mathcal{D}}}
$$

where $C_{\Lambda, u}$ is a constant depending on $\Lambda, u$ and $\Omega$ (more precisely of the $C^{2}$-norm of $u$ and the $C^{1}$-norm of $\Lambda$ ). Finally:

$$
\begin{gathered}
T_{4} \leq h_{\mathcal{D}} C_{\Lambda, u} \sum_{K \in \mathcal{T}} \sum_{\sigma \in \mathcal{E}_{K}}|\sigma|\left|\widetilde{I}_{\sigma}\left(v_{h_{\mathcal{D}}}\right)-v_{\sigma}\right| \\
\leq d^{1 / 2} h_{\mathcal{D}} C_{\Lambda, u}\left(\sum_{K \in \mathcal{T}} \sum_{\sigma \in \mathcal{E}_{K}} \frac{|\sigma| h_{K, \sigma}}{d}\right)^{\frac{1}{2}}\left(\sum_{K \in \mathcal{T}} \sum_{\sigma \in \mathcal{E}_{K}}|\sigma| h_{K, \sigma}\left(\frac{\widetilde{I}_{\sigma}\left(v_{h_{\mathcal{D}}}\right)-v_{\sigma}}{h_{K, \sigma}}\right)^{2}\right)^{\frac{1}{2}} \leq d^{1 / 2} h_{\mathcal{D}} C_{\Lambda, u}|\Omega|^{\frac{1}{2}}|| v_{h_{\mathcal{D}}} \mid h_{h_{\mathcal{D}}}
\end{gathered}
$$

Now, taking $v_{h_{\mathcal{D}}}=P_{\mathcal{D}}(u)-u_{h_{\mathcal{D}}}$, using (3.13) the coercivity of the scheme and the above results, we deduce that there exists $C>0$ depending on $u, \Lambda, d, \Omega$ and $\theta$ such that:

$$
\left\|P_{\mathcal{D}}(u)-u_{h_{\mathcal{D}}}\right\|_{h_{\mathcal{D}}} \leq C h_{\mathcal{D}}
$$

from which (3.11) immediately follows from the consistency of the semi hybrid gradients and the triangular inequality. Using Lemma 2.3, the triangular inequality and Theorem 2.4, we obtain (3.12).

This result would still hold true with less regularity assumptions: for instance, with $u \in H^{2}(\Omega)$ and $\Lambda \in$ $W^{1, \infty}\left(\Omega, \mathbb{M}_{d}(\mathbb{R})\right)$. However, the corresponding proof would be much more involved, and going from the regularity assumed in Theorem 3.4 to $u \in H^{2}(\Omega)$ and $\Lambda \in W^{1, \infty}\left(\Omega, \mathbb{M}_{d}(\mathbb{R})\right)$ is a quite classical problem, while the above proof already emphasizes the most important properties of our schemes, which are the reasons why we restricted ourselves to this simpler setting. While estimate (3.11) seems optimal regarding numerical results, as usual the $L^{2}$ estimate (3.12) is not sharp, and order 2 is obtained numerically (see Sect. 4). 


\section{EXAMPLES OF SEMI HYBRID SCHEMES}

In this section, we provide two ways of constructing semi hybrid schemes. The first one is solely based on always consistent numerical interpolation. The second one explains how the MPFA approximation ideas can be used.

\subsection{Barycentric interpolation}

For each $\sigma \in \mathcal{E}_{\text {int }}$, we define a barycentric interpolation of $\boldsymbol{x}_{\sigma}$, i.e. a family $\left(\left(\beta_{K}^{\sigma}\right)_{K \in \mathcal{T}},\left(\beta_{\sigma^{\prime}}^{\sigma}\right)_{\sigma^{\prime} \in \mathcal{E}_{\text {ext }}}\right)$ such that:

$$
\boldsymbol{x}_{\sigma}=\sum_{K \in \mathcal{T}} \beta_{K}^{\sigma} \boldsymbol{x}_{K}+\sum_{\sigma^{\prime} \in \mathcal{E}_{\mathrm{ext}}} \beta_{\sigma^{\prime}}^{\sigma} \boldsymbol{x}_{\sigma^{\prime}}
$$

and

$$
\sum_{K \in \mathcal{T}} \beta_{K}^{\sigma}+\sum_{\sigma^{\prime} \in \mathcal{E}_{\text {ext }}} \beta_{\sigma^{\prime}}^{\sigma}=1
$$

For any $K \in \mathcal{T}$, we denote by $\mathcal{N}_{K}$ the elements $L \in \mathcal{T}$ that shares a face or a node with $K$. We then assume that for any $\sigma \in \mathcal{E}_{\text {int }}, \beta_{L}^{\sigma}=0$ if $L \notin \mathcal{N}_{K}$, for every $K \in \mathcal{N}_{\sigma}, \beta_{\sigma^{\prime}}^{\sigma}=0$ if $\sigma^{\prime} \notin \mathcal{E}_{K}$, for every $K \in \mathcal{N}_{\sigma}$. Moreover, we assume that the number of non zero coefficients for each $\sigma \in \mathcal{E}_{\text {int }}$ is exactly $d+1$. Remark that it is always possible to find such a family of $d+1$ coefficients $\left(\left(\beta_{K}^{\sigma}\right)_{K \in \mathcal{T}},\left(\beta_{\sigma^{\prime}}^{\sigma}\right)_{\sigma^{\prime} \in \mathcal{E}_{\text {ext }}}\right)$, as the simplest polyhedron in dimension $d$ has at least $d+1$ faces, and thus at least $d+1$ exterior faces and neighbors.

Following the idea of [20], we define the barycentric interpolation $\left(I_{\sigma}(\cdot)\right)_{\sigma \in \mathcal{E}_{\text {int }}}$ associated to $\mathcal{D}$ by

$$
I_{\sigma}^{B}\left(u_{h}\right)=\sum_{K \in \mathcal{T}} \beta_{K}^{\sigma} u_{K}+\sum_{\sigma^{\prime} \in \mathcal{E}_{\text {ext }}} \beta_{\sigma^{\prime}}^{\sigma} u_{\sigma^{\prime}} \quad \forall \sigma \in \mathcal{E}_{\text {int }} .
$$

As this interpolation is exact on affine functions, it is clear that this interpolant is order 2 consistent on $C_{c}^{\infty}(\Omega)$.

\subsection{MPFA-O approximation}

We briefly recall the ideas of the MFPA-O approximation, introduced by Aavatsmark (see [1-3]). We denote $\mathcal{V}$ the set of vertices of the mesh, i.e. a family of points such that for any $K \in \mathcal{T}$, and any subset $H_{K}$ of $\mathcal{E}_{K}$ with $\operatorname{card}\left(H_{K}\right) \geq d$, then $\cap_{\sigma \in H_{K}}=\emptyset$ or $\cap_{\sigma \in H_{K}}=s$, for some $s \in \mathcal{V}$. For all $s \in \mathcal{V}$, we denote $\mathcal{E}_{s}$ the set $\{\sigma \in \mathcal{E} \mid s \in \sigma\}$, and $\mathcal{T}_{s}$ the set of cells $\{K \in \mathcal{T} \mid s \in \bar{K}\}$. For all $K \in \mathcal{T}$, the set $\mathcal{V}_{K}$ stands for $\left\{s \in \mathcal{V} \mid K \in \mathcal{T}_{s}\right\}$, while for all $\sigma \in \mathcal{E}$, the set $\mathcal{V}_{\sigma}\left\{s \in \mathcal{V} \mid \sigma \in \mathcal{E}_{s}\right\}$.

In dimension 2, for any $s \in \mathcal{V}$ and any $\sigma \in \mathcal{V}_{s}, \sigma_{s}$ denotes the subset of $\sigma$ defined as the interval between $s$ and $\boldsymbol{x}_{\sigma}$, and we denote $\boldsymbol{x}_{\sigma_{s}}$ the center of $\sigma_{s}$. In dimension 3 for any $s \in \mathcal{V}$ and any $\sigma \in \mathcal{V}_{s}, \sigma_{s}$ denotes the the open set constructed from $s, \boldsymbol{x}_{\sigma}$, and the center of the edges of $\sigma$ that contains $s$.

The volume $K_{s}$ is then defined, for any $K \in \mathcal{T}_{s}$, as the union over $\sigma \in \mathcal{E}_{K} \cap \mathcal{E}_{s}$ of the cones with basis $\sigma_{s}$ and summit $\boldsymbol{x}_{K}$. In each $K_{s}$, a discrete gradient $\nabla_{K}^{s}$ is defined by

$$
\nabla_{K}^{s}\left(\left(u_{M}\right)_{M \in \mathcal{T}_{s}},\left(u_{\mu_{s}}\right)_{\mu \in \mathcal{E}_{s}}\right)=\sum_{\sigma \in \mathcal{E}_{K} \cap \mathcal{E}_{s}}\left(u_{\sigma_{s}}-u_{K}\right) \boldsymbol{g}_{K, \sigma, s},
$$

where the $\boldsymbol{g}_{K, \sigma, s}$ satisfy:

$$
\sum_{\sigma \in \mathcal{E}_{K} \cap \mathcal{E}_{s}} \boldsymbol{g}_{K, \sigma, s}\left(\boldsymbol{x}_{\sigma_{s}}-\boldsymbol{x}_{K}\right)^{T}=I_{d}
$$

which ensures that it is exact on affine functions in $K_{s}$. In dimension 2, it suffices to take the gradient of the $P 1$ interpolation between $\boldsymbol{x}_{K}$ and $\left(\boldsymbol{x}_{\sigma_{s}}\right)_{\sigma \in \mathcal{E}_{s}}$ to obtain appropriate values for the $\boldsymbol{g}_{K, \sigma, s}$, and retrieve the classical MPFA-O scheme of [2]. We refer the reader to $[2,4]$ for constructions of the $\boldsymbol{g}_{K, \sigma, s}$ in higher dimensions. 
For each $s \in \mathcal{V}$, the MPFA-O partial interpolants are defined as the solution $\left(u_{\sigma_{s}}\right)_{\sigma \in \mathcal{E}_{s}}$, of the following system:

$$
\Lambda_{K} \nabla_{K}^{s}\left(\left(u_{M}\right)_{M \in \mathcal{T}_{s}},\left(u_{\mu_{s}}\right)_{\mu \in \mathcal{E}_{s}}\right) \cdot \boldsymbol{n}_{K, \sigma}+\Lambda_{L} \nabla_{L}^{s}\left(\left(u_{M}\right)_{M \in \mathcal{T}_{s}},\left(u_{\mu_{s}}\right)_{\mu \in \mathcal{E}_{s}}\right) \cdot \boldsymbol{n}_{L, \sigma}=0 \quad \forall \sigma \in \mathcal{E}_{s}, \mathcal{N}_{\sigma}=\{K, L\} .
$$

Thus, the $\left(u_{\sigma_{s}}\right)_{\sigma \in \mathcal{E}_{s}}$ can be seen as functions of $\left(u_{M}\right)_{M \in \mathcal{T}_{s}}$. The MPFA-O partial fluxes are defined as:

$$
F_{K, \sigma_{s}}^{O}\left(\left(u_{M}\right)_{M \in \mathcal{T}_{s}}\right)=\left|\sigma_{s}\right| \Lambda_{K} \nabla_{K}^{s}\left(\left(u_{M}\right)_{M \in \mathcal{T}_{s}},\left(u_{\mu_{s}}\right)_{\mu \in \mathcal{E}_{s}}\right) \cdot \boldsymbol{n}_{K, \sigma}
$$

and are consequently also functions of $\left(u_{M}\right)_{M \in \mathcal{T}_{s}}$. Then, the MPFA-O interpolant is defined as:

$$
I_{\sigma}^{O}\left(u_{\mathcal{T}}\right)=\sum_{s \in \mathcal{V}_{\sigma}} \frac{\left|\sigma_{s}\right|}{|\sigma|} u_{\sigma_{s}}\left(\left(u_{M}\right)_{M \in \mathcal{T}_{s}}\right)
$$

where $u_{\mathcal{T}}=\left(u_{K}\right)_{K \in \mathcal{T}}$ while the MPFA flux are defined as

$$
F_{K, \sigma}^{M P F A-O}\left(u_{\mathcal{T}}\right)=\sum_{s \in \mathcal{V}_{\sigma}} F_{K, \sigma_{s}}^{O}\left(\left(u_{M}\right)_{M \in \mathcal{T}_{s}}\right)
$$

As this construction is exact for piecewise affine functions of $\mathcal{Q}_{\Lambda}$, consistency of both flux and interpolants can be established (see [4,5], or [1-3]). Notice that both interpolants and flux only involves the neighbors, by vertex or face, of the considered cell $K$, thus giving compact stencil expressions.

\subsection{Gradient seeds}

Considering the fact that we wish to show that it is possible obtain precise schemes for which face unknowns can be eliminated, we will consider gradient seeds that involves cell unknowns only. We define two gradient seeds:

- The "barycentric" gradient seed:

$$
\nabla_{K}^{S}\left(u_{h}\right)=\sum_{\sigma^{\prime} \in \mathcal{E}_{K} \cap \mathcal{E}_{\mathrm{int}}} \frac{\left|\sigma^{\prime}\right|}{|K|}\left(I_{\sigma^{\prime}}^{B}\left(u_{h}\right)-u_{K}\right) \boldsymbol{n}_{K, \sigma^{\prime}}+\sum_{\sigma^{\prime} \in \mathcal{E}_{K} \cap \mathcal{E}_{\text {ext }}} \frac{\left|\sigma^{\prime}\right|}{|K|}\left(u_{\sigma^{\prime}}-u_{K}\right) \boldsymbol{n}_{K, \sigma^{\prime}} \quad \forall K \in \mathcal{T},
$$

where $\left(I_{\sigma}\right)_{\sigma \in \mathcal{E}_{\text {int }}}^{B}$ denotes the family of barycentric interpolators associated to $\mathcal{D}$. Consistency of this gradient can easily be proved using Taylor's expansion (see for instance in [19]).

- The "MPFA O" gradient seed:

$$
\nabla_{K}^{S}\left(u_{h}\right)=\frac{1}{|K|} \sum_{\sigma^{\prime} \in \mathcal{E}_{K}} F_{K, \sigma^{\prime}}^{M P F A-O}\left(u_{h}\right) \Lambda_{K}^{-1}\left(\boldsymbol{x}_{\sigma^{\prime}}-\boldsymbol{x}_{K}\right) \quad \forall K \in \mathcal{T},
$$

where $\Lambda_{K}=\frac{1}{|K|} \int_{K} \Lambda$ and $F_{K, \sigma^{\prime}}^{M P A-O}\left(u_{h}\right)$ is the multi-point flux approximation of the flux through $\sigma$ from $K$, given by the $\mathrm{O}$-scheme. The strong consistency of such reconstructed gradient on the space $\mathcal{Q}_{\Lambda}$ was proved in [5], to which we refer the reader.

\subsection{Semi hybrid schemes}

We will consider four semi hybrid schemes in our numerical exploration of their properties:

(i) The "Semi Hybrid Hybrid-Barycentric Gradient" (SH-HB) method: we take $\mathcal{E}_{\mathcal{F}}=\mathcal{E}$, and use the barycentric gradient seed.

(ii) The "Semi Hybrid Barycentric interpolation-Barycentric Gradient" (SH-BB) method: we take $\mathcal{E}_{\mathcal{I}}=\mathcal{E}_{\text {int }}$, use the barycentric interpolator and the barycentric gradient seed. 

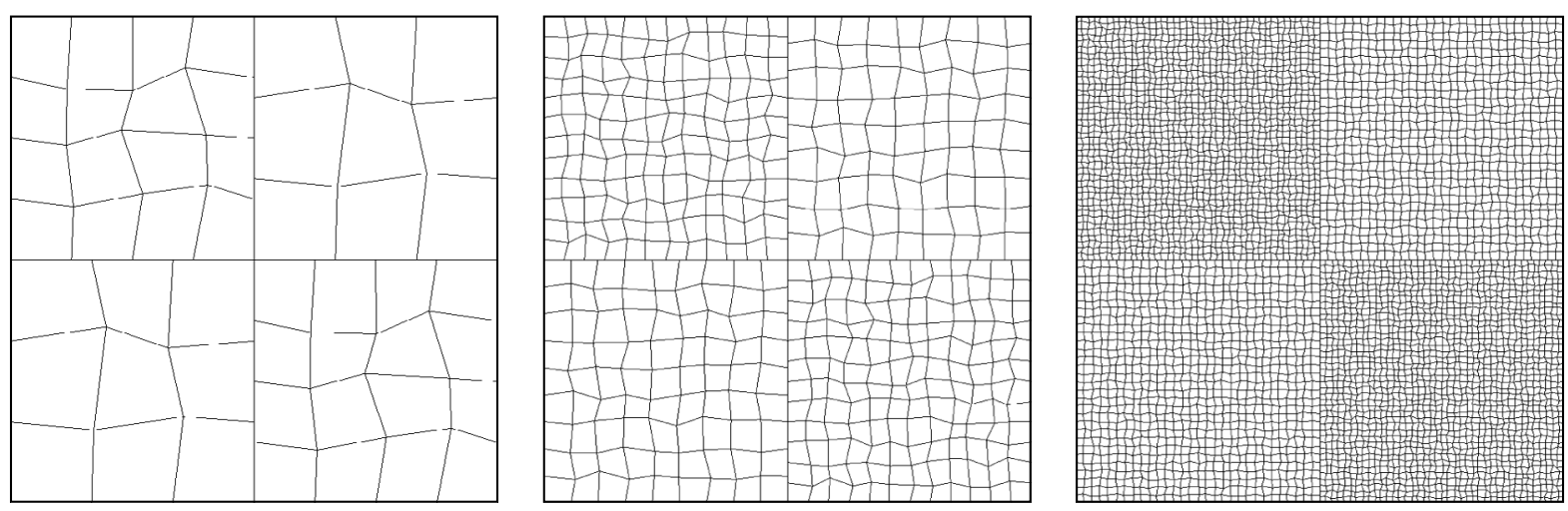

FiguRE 1. Unconforming meshes.

(iii) The "Semi Hybrid Hybrid-MPFA-O Gradient" (SH-HO) method: we take $\mathcal{E}_{\mathcal{F}}=\mathcal{E}$, and use the MPFA-O gradient seed.

(iv) The "Semi Hybrid MPFA-O interpolation-MPFA-O Gradient" (SH-OO) method: we take $\mathcal{E}_{\mathcal{I}}=\mathcal{E}_{\text {int }}$, use the MPFA-O interpolator and the MPFA-O gradient seed.

For the stabilization term, we always use $S_{K, \sigma}=\Lambda_{K, \sigma}$. The four schemes allow to eliminate interior face unknowns (when present). Their stencil, when keeping face unknowns, is for a given cell $K$ included in the set of neighbors (by vertex or face) and neighbors of neighbors of the cell $K$. Thus it gives relatively compact expressions. However, when eliminating face unknowns for the SH-HB and SH-HO methods, the resulting fully cell-centered schemes have larger stencils. This means that interpolation allows to obtain cell-centered schemes with smaller stencils, which is one of the main interests of using interpolation rather than face unknowns.

\section{NumericAl RESULtS}

We now present some numerical results in dimension 2. For all the numerical tests, each $\boldsymbol{x}_{K}$ is chosen as the center of the cell $K$. To serve as reference, we use the classical hybrid scheme, in the [19] version (i.e. with $\sqrt{d}$ in the stabilization term) and the classical finite volume MPFA-O scheme.

\subsection{Convergence tests with homogeneous anisotropic tensors}

Throughout this subsection, we consider the function:

$$
u(\boldsymbol{x})=\sin \left(\frac{m \pi x_{1}}{L_{1}}\right) \sin \left(\frac{n \pi x_{2}}{L_{2}}\right)
$$

on the domain $] 0, L_{1}[\times] 0, L_{2}$ [, for several values of the integers $m$ and $n$, with an homogeneous anisotropic tensor $\Lambda$ given by:

$$
\Lambda=\left(\begin{array}{cc}
1.5 e 2 & -0.5 \\
-0.5 & 1.5 .
\end{array}\right)
$$

The source term $f$ is then analytically computed. We consider three test cases: Test $1:(m, n)=(1,1)$, Test 2 : $(m, n)=(7,7)$, Test 3: $(m, n)=(12,1)$. We use nonconforming meshes, generated through the fusion of four randomly perturbed cartesian meshes of $] 0, \frac{L_{1}}{2}[\times] 0, \frac{L_{2}}{2}[,] \frac{L_{1}}{2}, L_{1}[\times] 0, \frac{L_{2}}{2}[] 0,, \frac{L_{1}}{2}[\times] \frac{L_{2}}{2}, L_{2}[$ and $] \frac{L_{1}}{2}, L_{1}[\times] \frac{L_{2}}{2}, L_{2}[$. For the first and fourth quadrants, we use a $3 k \times 3 k$ cartesian mesh, and a $4 k \times 4 k$ for the second and the third ones, and we refine by increasing $k$. Those meshes are illustrated in Figure 1. 

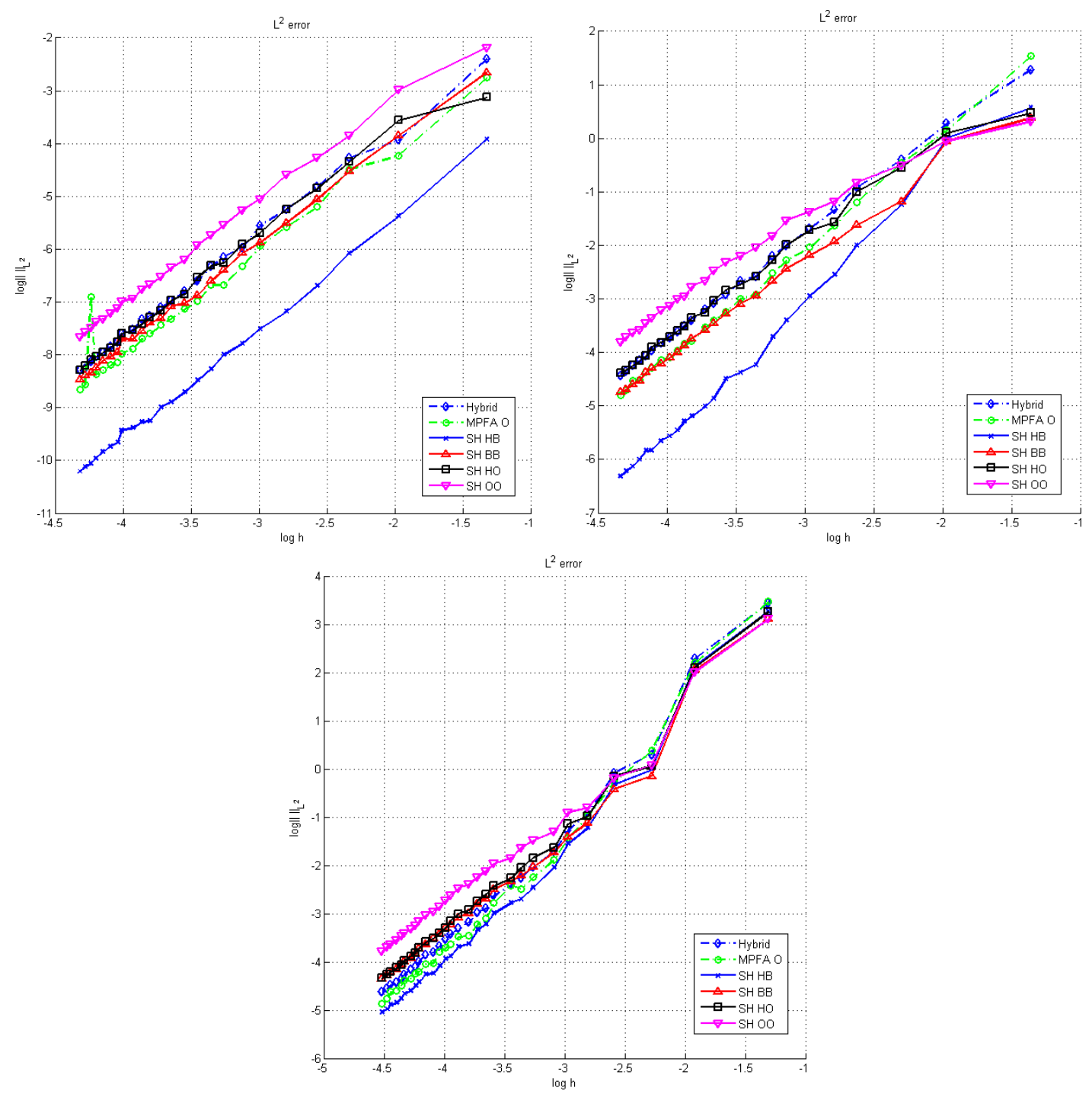

FiguRE 2. Convergence rates on unconforming meshes, for the three tests cases, relative $L^{2}$ error.

We now present the errors corresponding to estimates (3.12) in Figure 2. The approximate convergence rates, obtained by a linear fitting of the asymptotic part of the convergence curves, are regrouped in the following table:

\begin{tabular}{lccc}
\hline \multirow{2}{*}{ Scheme } & Test 1 & Test 2 & Test 3 \\
\cline { 2 - 4 } & $\|\cdot\|_{L^{2}}$ & $\|\cdot\|_{L^{2}}$ & $\|\cdot\|_{L^{2}}$ \\
\hline Hybrid & 2.1343 & 1.9795 & 2.0448 \\
MPFA-O & 2.0137 & 2.0149 & 2.0320 \\
SH-HB & 2.2055 & 2.1338 & 2.0186 \\
SH-BB & 2.0854 & 1.9258 & 1.9777 \\
SH-HO & 2.0338 & 1.9824 & 2.0192 \\
SH-OO & 2.1033 & 1.9495 & 1.9740 \\
\hline
\end{tabular}



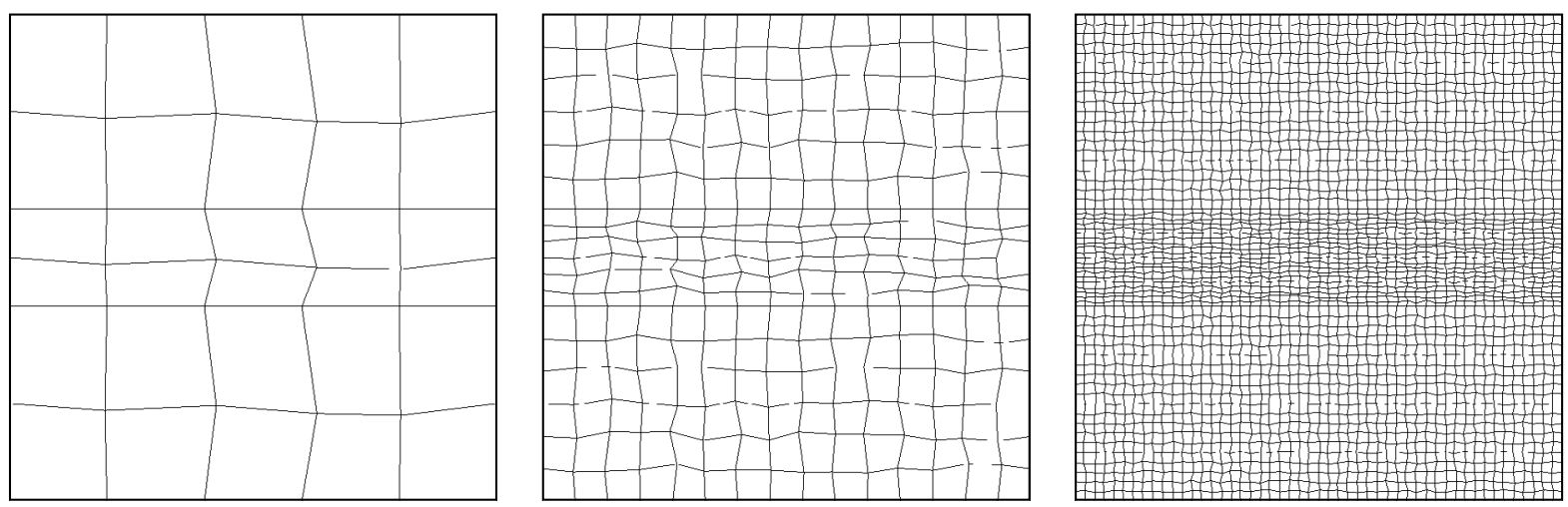

Figure 3. Meshes adapted to the heterogeneity.

First, observe that all schemes are convergent for the three test cases, confirming the ability to handle nonconformity of the semi hybrid method. Moreover, all schemes achieve convergence rates close to 2, which shows that (3.12) is suboptimal. The classical MPFA O-method exhibits some instability, due to its non coercivity and the anisotropy of the problem, while all semi hybrid schemes have a behavior similar to the classical hybrid scheme.

\subsection{Heterogeneous tensors}

We now assume that the domain $\Omega$ is divided into two subdomains $\Omega_{1}$ and $\Omega_{2}$, such that:

$$
\Lambda_{\Omega_{\Omega_{1}}}=I_{d} \quad \text { and } \quad \Lambda_{\Omega_{2}}=10^{-4} I_{d}
$$

The domains $\Omega_{1}$ and $\Omega_{2}$ are defined as follows:

$$
\left.\Omega_{2}=\right] 0, L_{1}[\times] 2 L_{2} / 5,3 L_{2} / 5\left[\quad \text { and } \quad \Omega_{1}=\Omega \backslash \overline{\Omega_{2}} .\right.
$$

We use conforming meshes generated through the fusion of randomly cartesian meshes of $\Omega_{2}$ and the two connected parts of $\Omega_{1}$, as described in Figure 3. We consider the analytic solution:

$$
u(x, y)=\alpha(x, y) x(x-1)(y-1)\left(y-\frac{2 L_{2}}{5}\right)\left(y-\frac{3 L_{2}}{5}\right)
$$

where $\alpha=1$ in $\Omega_{1}$, and $\alpha=1 e 2$ in $\Omega_{2}$. Thus, $u \in H_{0}^{1}(\Omega)$ and $\nabla u \cdot e_{y}$ is continuous at $y=\frac{2 L_{2}}{5}$ and $y=\frac{3 L_{2}}{5}$. However, $u$ does not belong to $H^{2}(\bar{\Omega})$ (or $C^{2}(\bar{\Omega})$ ) because of the discontinuities of the gradient. We present the $L^{2}$ error on the solution and its gradient in Figure 4 . The first observation is that all the schemes are still convergent in this situation. However, we see that the precision of the schemes that uses barycentric interpolation or barycentric gradients severely diminishes in this situation, giving poor approximations, while the hybrid, MPFA O, SH-HO and $\mathrm{SH}-\mathrm{OO}$ schemes remain rapidly convergent. The $\mathrm{SH}-\mathrm{OO}$ error level is close to the hybrid scheme one, while the $\mathrm{SH}-\mathrm{HO}$ is, on this particular example, even more precise. The additional precision with respect to the $\mathrm{SH}-\mathrm{OO}$ scheme comes most certainly from the fact that the $\mathrm{SH}-\mathrm{HO}$ method uses face unknowns in its formulation (even if they are eliminated in practice), while the SH-OO uses numerical interpolation. As face unknowns elimination enlarges the stencil, the choice between $\mathrm{SH}-\mathrm{HO}$ and $\mathrm{SH}-\mathrm{OO}$ is consequently a trade-off between precision and matrix fill-in. On the other hand, the MPFA-O gradient takes into account the heterogeneity, which explains the additional precision with respect to the hybrid scheme.

To illustrate this trade off, we now look at the matrices underlying our linear systems. In the above experiments, a simple LU factorization has been used for solving the linear systems. However for large scale problems, 


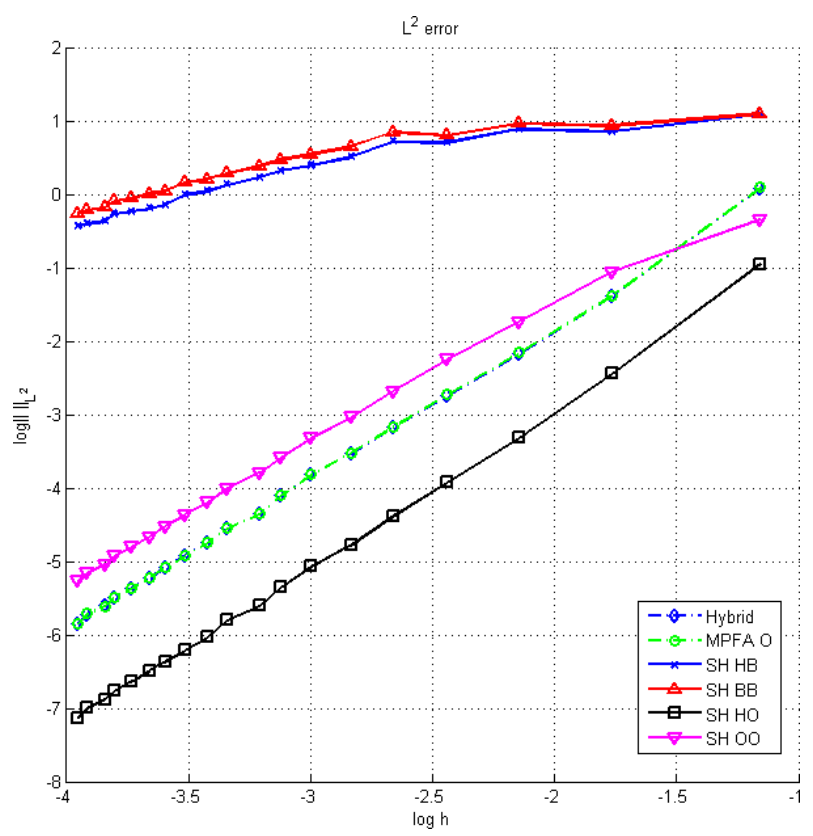

FiguRE 4. Convergence curves for the heterogeneous test case, $L^{2}$ error on the solution.

iterative solvers are usually favored, combined with a well-suited preconditioning technique. We use the an ILU(0) factorization combined with a reverse Cuthill-McKee reordering as preconditioner, and a BiCGstab solver. We display on the next table the number of degrees of freedom of each system, the maximum number of entries on a line of the matrix (the maximum stencil size), the number of non-zero entries in the matrix, the number of non-zero entries of the $L$ an $U$ part of the incomplete $L U$ factorization, and finally the number of iterations of the linear solver. For simplicity, we do not explicitly eliminate face unknowns for the SH-HO scheme. For the heterogeneous test case, we obtain the following results, for three different mesh sizes, considering only schemes that can handle correctly heterogeneities:

\begin{tabular}{lcccccc}
\hline \multicolumn{7}{c}{ Mesh 1 } \\
\hline Scheme & Number of dofs & Max stencil size & nnz & nnz $(\mathrm{L})$ & nnz(U) & BiCGstab iterations \\
\hline Hybrid & 1484 & 9 & 10388 & 5936 & 5936 & 16 \\
MPFA-O & 568 & 9 & 4048 & 2358 & 2358 & 8 \\
SH-HO & 1484 & 49 & 32520 & 17002 & 17002 & 28 \\
SH-OO & 568 & 25 & 10804 & 5686 & 5686 & 6 \\
\hline \multicolumn{7}{c}{ Mesh 2} \\
\hline Scheme & Number of dofs & Max stencil size & nnz & nnz $(\mathrm{L})$ & nnz $(\mathrm{U})$ & BiCGstab iterations \\
\hline Hybrid & 7628 & 9 & 54413 & 30901 & 30901 & 31 \\
MPFA-O & 2628 & 9 & 21478 & 12053 & 12053 & 14 \\
SH-HO & 7628 & 49 & 174315 & 90852 & 90852 & 168 \\
SH-OO & 2628 & 25 & 58014 & 30321 & 30321 & 12 \\
\hline \multicolumn{7}{c}{ Mesh 3 } \\
\hline Scheme & Number of dofs & Max stencil size & nnz & nnz(L) & nnz(U) & BiCGstab iterations \\
\hline Hybrid & 17794 & 9 & 132938 & 75366 & 75366 & 48 \\
MPFA-O & 6188 & 9 & 52308 & 29248 & 29248 & 25 \\
SH-HO & 17794 & 49 & 428610 & 223202 & 223202 & 638 \\
SH-OO & 6188 & 25 & 142724 & 74456 & 74456 & 20 \\
\hline
\end{tabular}


We see on these tables that the relative cost of each method is not easy to measure: the SH-OO scheme and the Hybrid scheme have a similar number of non-zero entries in the matrix, or in the $L$ and $U$ factors. Thus, any matrix product will cost approximately the same for these two schemes, despite the fact that the number of degrees of freedom (dofs) and the stencil of the schemes are very different. Moreover the BiCGstab algorithm converges faster for the SH-OO scheme than for the Hybrid scheme. Thus, the cost of the two schemes is of the same order, and is slightly better of the SH-OO scheme in this particular case. We also clearly recover the fact that the $\mathrm{SH}-\mathrm{HO}$ scheme is more costly than the SH-OO, as it exhibits larger stencils, a greater number of non-zero entries, and a considerably higher number of linear solver iterations. This reveals that other preconditioners, and probably a better reordering, should probably be considered and investigated for the SH-HO scheme. However, it remains the most precise scheme for our heterogeneous test case. Varying the mesh size confirm these results. This shows that semi hybrid schemes can indeed be competitive with respect to the hybrid and MPFA O scheme, despite their enlarged stencil. Remark that these experiments illustrate the fact that the number of unknowns or the size of the stencil is not necessarily a good measure of the overall cost of a method.

Of course, one of the most interesting features of the semi hybrid method is that it allows to mix all these approaches, by choosing carefully the most suited gradient seed and interpolant, cell by cell and face by face. For instance, as in the SUSHI approach, one could use face unknowns at the heterogeneous interface, and the MPFA-O based gradient seed in the surrounding cells, while using barycentric interpolation and barycentric based gradient seed where the tensor is smooth enough. This would give, once face unknowns eliminated, a cell-centered, symmetric coercive and convergent method, with a relatively compact stencil. Remark that any other consistent MPFA methods, such as the L-method or the G-method (see [5]) could have been used instead of the O-method, and would also produce coercive and convergent schemes, while allowing to narrow the stencil.

The fact that we can mix all the approaches is particularly relevant in the context of parallel computing when using a domain partionning technique. As a matter of fact, schemes with large stencils can increase communication time, and thus slow the execution of parallel codes. However, as the semi hybrid method allows for a simple and dynamic switching between the schemes in any part of the domain, this problem can easily be overcome. To get the smallest number of communications, for faces which are interfaces between two sub-domains of the domain partionning algorithm, one can simply keep the face unknowns and use the hybrid gradient seed in the surrounding cells. Elsewhere in the sub-domains, one can use any gradient seed. The resulting scheme inherits the good parallel capabilities of the original hybrid method, while allowing inside the sub-domains to benefit from the properties of the semi-hybrid schemes. If one wishes to use other gradients or face interpolation at the boundaries of the subdomains, the gradient seed as well as the interpolant should be communicated, resulting in much heavier communications. The choice is mainly a question of trade-off between the number of communications and the resulting properties, in terms of size and number of non-zero entries, of the resulting linear system.

\section{Conclusion}

A new family of symmetric, always coercive discretization schemes for anisotropic and heterogeneous diffusion problems was presented. They allow to use a wide class of consistent approximations of the gradient operator, and give birth to a large class of schemes. In particular, for gradients which do not use interior face unknowns, cellcentered schemes are obtained. Convergence to minimal regularity solution enters a well understood framework, and error estimates for generic consistent gradients were obtained under suitable regularity assumptions. The numerical results shows the good behavior of the schemes. The methods are flexible and adaptive, as different discrete gradients can be used in any part of the domain.

Acknowledgements. The author would like to thank L. Agélas for our numerous discussions on the subject of this article. 


\section{REFERENCES}

[1] I. Aavatsmark, T. Barkve, O. Boe and T. Mannseth, Discretization on non-orthogonal, quadrilateral grids for inhomogeneous, anisotropic media. J. Comput. Phys. 127 (1996) 2-14.

[2] I. Aavatsmark, T. Barkve, O. Boe and T. Mannseth, Discretization on unstructured grids for inhomogeneous, anisotropic media part i: Derivation of the methods. SIAM J. Sci. Comput. 19 (1998a) 1700-1716.

[3] I. Aavatsmark, T. Barkve, O. Boe and T. Mannseth, Discretization on unstructured grids for inhomogeneous, anisotropic media. part ii: Discussion and numerical results. SIAM J. Sci. Comput. 19 (1998b) 1717-1736.

[4] L. Agélas and R. Masson, Convergence of finite volume mpfa o type schemes for heterogeenous anisotropic diffusion problems on general meshes. C.R. Acad. Paris Ser. I 346 (2008).

[5] L. Agélas, D.A. Di Pietro and J. Droniou, The g method for heterogeneous anisotropic diffusion on general meshes. ESAIM: M2AN 11 (2010) 597-625.

[6] L. Agélas, D.A. Di Pietro, R. Eymard and R. Masson, An abstract analysis framework for nonconforming approximations of anistropic heterogeneous diffusion problems. IJFV International Journal On Finite Volumes 7 (2010).

[7] L. Agélas, D.A. Di Pietro and R. Masson, A symmetric and coercive finite volume scheme for multiphase porous media flow problems with applications in the oil industry. In Finite volume for Complex Applications V. Edited by R. Eymard ans J.-M. Hérard. Wiley (2008) 35-51.

[8] L. Beirao da Veiga, F. Brezzi, A. Cangiani, G. Manzini, L.D. Marini and A. Russo, Basic principles of virtual element methods. Math. Models Methods Appl. Sci. 23 (2013) 199-214.

[9] L. Beirao da Veiga, K. Lipnikov and G. Manzini, The Mimetic Finite Difference Method for Elliptic Problems. Springer (2014).

[10] F. Brezzi and M. Fortin, Mixed and Hybrid Finite Element Methods. Springer-Verlag, New York (1991).

[11] F. Brezzi, K. Lipnikov and M. Shashkov, Convergence of the mimetic finite difference method for diffusion problems on polyhedral meshes. SIAM J. Numer. Anal. 43 (2005a) 1872-1896.

[12] F. Brezzi, K. Lipnikov and V. Simoncini, A family of mimetic finite difference methods on polygonal and polyhedral meshes. Math. Models Methods Appl. Sci. 15 (2005b) 1533-1551.

[13] J. Droniou, Finite volume schemes for diffusion equations: introduction to and review of modern methods. Special edition "P.D.E. Discretizations on Polygonal Meshes". M3AS 24 (2014) 1575-1619.

[14] J. Droniou and R. Eymard, A mixed finite volume scheme for anisotropic diffusion problems on any grid. Numer. Math. 105 (2006) 35-71.

[15] J. Droniou, R. Eymard, T. Gallouët and R. Herbin, A unified approach to mimetic finite differences, hybrid finite volume and mixed finite volume methods. IMA J. Numer. Anal. 31 (2011) 1357-1401.

[16] J. Droniou, R. Eymard, T. Gallouët and R. Herbin, Gradient schemes: a generic framework for the discretisation of linear, nonlinear and nonlocal elliptic and parabolic equations. M3AS 23 (2013) 2395-2432.

[17] R. Eymard, T. Gallouët and R. Herbin, Finite volume methods, In Techniques of scientific computiing, Part III. Handb. Numer. Anal. Edited by P.G. Ciarlet and J.-L. Lions. North-Holland, Amsterdam (2000) 713-1020.

[18] R. Eymard, T. Gallouët and R. Herbin, A new finite volume scheme for anisotropic diffusion problems on general grids: convergence analysis. C.R. Math. Acad. Sci. Paris 344 (2007a) 403-406.

[19] R. Eymard, T. Gallouët and R. Herbin, Discretisation of heterogeneous and anisotropic diffusion problems on general nonconforming meshes sushi: a scheme using stabilisation and hybrid interfaces. IMA J. Numer. Anal. 30 (2010) 1009-1043x.

[20] R. Eymard and R. Herbin, A new colocated finite volume scheme for the incompressible navier-stokes equations on general non matching grids. C.R. Math. Acad. Sci. Paris 344 (2007b) 659-662.

[21] R. Eymard, R. Herbin and C. Guichard, Small stencil 3d schemes for diffusive flows in porous media. ESAIM: M2AN 46 (2012) 265-290.

[22] D.A. Di Pietro, Cell centered galerkin methods. C.R. Acad. Sci. Paris Ser. I 348 (2010) 31-34.

[23] D.A. Di Pietro, Cell centered galerkin methods for diffusive problems. ESAIM: M2AN 46 (2012) 111-144.

[24] M. Vohralík, Equivalence between lowest-order mixed finite element and multi-point finite volume methods on simplicial meshes. ESAIM: M2AN 40 (2006) 367-391.

[25] M. Vohralík and B. Wohlmuth, From face to element unknowns by local static condensation with application to nonconforming finite elements. Comput. Methods Appl. Mech. Eng. 253 (2013a) 517-529.

[26] M. Vohralík and B. Wohlmuth, Mixed finite element methods: implementation with one unknown per element, local flux expressions, positivity, polygonal meshes, and relations to other methods. Math. Models Methods Appl. Sci. 23 (2013b) 803-838. 